OPEN ACCESS

Edited by:

Philippe Vernoux

UMR5256 Institut de Recherches sur la Catalyse et l'Environnement de Lyon (IRCELYON), France

Reviewed by:

Valerie Meille

UMR5285 Laboratoire de Génie des Procédés Catalytiques (LGPC), France

Yiqian Wan,

Sun Yat-sen University, China

*Correspondence: Filippo Maria Perna filippo.perna@uniba.it Vito Capriati vito.capriati@uniba.it

Specialty section: This article was submitted to Green and Sustainable Chemistry, a section of the journa Frontiers in Chemistry

Received: 25 June 2019 Accepted: 10 October 2019 Published: 30 October 2019

Citation:

Quivelli AF, Vitale P, Perna FM and Capriati V (2019) Reshaping Ullmann Amine Synthesis in Deep Eutectic

Solvents: A Mild Approach for

Cu-Catalyzed C-N Coupling Reactions With No Additional Ligands.

Front. Chem. 7:723.

doi: 10.3389/fchem.2019.00723

\section{Reshaping Ullmann Amine Synthesis in Deep Eutectic Solvents: A Mild Approach for Cu-Catalyzed C-N Coupling Reactions With No Additional Ligands}

\author{
Andrea Francesca Quivelli, Paola Vitale, Filippo Maria Perna* and Vito Capriati* \\ Dipartimento di Farmacia-Scienze del Farmaco, Università di Bari "Aldo Moro", Consorzio C.I.N.M.P.I.S., Bari, Italy
}

The Cul-catalyzed Ullmann amine cross-coupling between (hetero)aryl halides (Br, I) and aromatic and aliphatic amines has been accomplished in deep eutectic solvents as environmentally benign and recycling reaction media. Under optimized conditions, the reaction proceeds smoothly under mild conditions $\left(60-100^{\circ} \mathrm{C}\right)$ in air, in the absence of ligands, with a catalyst (Cul) loading of 10 mol\% and $\mathrm{K}_{2} \mathrm{CO}_{3}$ (aliphatic primary and secondary amines) or $t$-BuOK (aromatic amines) as the base. A variety of amines have been synthesized in yields up to $98 \%$ with a broad substrate scope.

Keywords: Ullmann amination reaction, copper catalysis, deep eutectic solvents, amine synthesis, green chemistry

\section{INTRODUCTION}

The century "classical" Ullmann reaction is known to be the first transition metal-mediated organic transformation, and it was used for the synthesis of symmetric biaryls from aryl halides (Ullmann, 1901, 1903, 1904; Goldberg, 1906). However, as it suffered from the requirement of stoichiometric amounts of copper salts, harsh reaction conditions (temperatures $\geq 200^{\circ} \mathrm{C}$ ), poor functional group tolerance and low yields of the expected adducts, it remained dormant for decades with limited applications. As a result of the tremendous impact of Pd-catalyzed cross-coupling reactions have had in the field of organic synthesis over the past 25 years (Seechurn et al., 2012; Sherwood et al., 2019), there has been recently a revival of interest in the so-called "Ullmann-type" reaction, which makes use of cheaper and environmentally responsible first-row transition metals to catalyze the nucleophilic aromatic substitution between an aryl halide and a nucleophile (Cristau et al., 2004, 2005; Chen and Chen, 2006; Shafir and Buchwald, 2006; Jones et al., 2007; Ma and Cai, 2008; Tye et al., 2008; Beletskaya and Ananikov, 2011; Sambiagio et al., 2014). The fundamental interrogation of how this reaction proceeds (radical or polar mechanism) has been a matter of debate throughout the years, and details still remain challenging today. A mechanism involving a $\mathrm{Cu}(\mathrm{I}) / \mathrm{Cu}(\mathrm{III})$ catalytic cycle has been experimentally supported in most Ullmann-type couplings, whereas the one-electron redox pathway involving aryl radical species is thought to take place in certain C-Heteroatom coupling reactions under light irradiation (Strieter et al., 2005; Tye et al., 2008; Giri and Hartwig, 2010; Sperotto et al., 2010; Casitas and Ribas, 2013; Ribas and Güell, 2014).

Among $\mathrm{N}$-arylation strategies available in the armory of the synthetic chemist, the Ullmann amine synthesis (UAS) has sparked in recent years the interest of the scientific community as it 
well complements the most famous Pd-catalyzed HartwigBuchwald amination reaction (Hartwig, 1998, 2000; Wolfe et al., 1998; De Meijere and Diederich, 2004; Surry and Buchwald, 2008). Key aspects of the UAS are the use of (a) polydentate auxiliary ligands with oxygen- or nitrogen coordination sites (e.g., diamines, amino acids, oximes, diols, 1,10-phenantrolines), which often improve the solubility of the copper precursors and the stability of the active catalyst, and/or (b) toxic and volatile organic compounds (VOCs) (e.g., DMF, DMSO, dioxane, THF, toluene) (Scheme 1A) (Evano et al., 2008; Monnier and Taillefer, 2009; Surry and Buchwald, 2010; Zhang et al., 2012b; Jiang et al., 2013; Zhou et al., 2015; Gao et al., 2017). Copper-catalyzed $N$ arylation in water or in aqueous media are also known. However, they are usually carried out in the presence of phase transfer catalysts and with ligands, and often require high reaction temperature $\left(100-130^{\circ} \mathrm{C}\right.$ ) ( $\mathrm{Li}$ et al., 2010; Huang et al., 2013; Sambiagio et al., 2014; Chakraborti et al., 2018). Interestingly, Wei et al. have reported in 2011 the copper powder-catalyzed ligand-free amination of aryl halides with aliphatic amines in neat water and in air at $100^{\circ} \mathrm{C}$, but secondary amines and aniline derivatives proved to be not reactive under these conditions (Jiao et al., 2011). Recent publications have highlighted the successful use of (engineered) surfactants (a) as ligands to stabilize the copper intermediates in the catalytic transformation and (b) to provide a micellar environment favoring the aggregation of reagents in water (Liu and Zhou, 2013; Ge et al., 2019). Green pathways to biaryls via palladium nanoparticles-catalyzed Ullmann reactions in ionic liquids or in ionic liquids/supercritical carbon dioxide systems have also been reported (Durán Pachón et al., 2006; Calò et al., 2009; Cheng et al., 2010).

Growing efforts are continuously being made to replace harmful, carcinogenic, petroleum-based VOCs with "greener," recyclable neoteric reaction media, as the largest contribution to the waste stream (over $80 \%$ ) in the practice of synthetic organic chemistry is provided by solvents (Lipshutz and Ghorai, 2014; Lipshutz et al., 2016). As for the Ullmann-type reaction, an important step forward has recently been made by Vaccaro et al. who developed a waste-minimized protocol for coppercatalyzed UAS in the biomass-derived furfuryl alcohol able to act as an effective bidentate ligand for copper and also to form an azeotrope with water (Scheme 1B) (Ferlin et al., 2018). Deep Eutectic Solvents (DESs) are mixtures usually made up of a hydrogen bond donor (HBD) and a hydrogen bond acceptor (HBA) that, upon mixing together in a proper molar ratio and heating, show an eutectic point temperature far below that of an ideal liquid mixture because of strong hydrogen-bonding interactions between the individual components that decreases the lattice energy of the system. DESs have been defined as the organic reaction medium of the twenty-first century and provide a valuable "green" alternative to common VOCs because of some interesting properties: a low vapor pressure, thermal stability, non-flammability and a high heat capacity (Ruß and König, 2012; Zhang et al., 2012a; García-Álvarez, 2015; GarcíaÁlvarez et al., 2015, 2018; Liu et al., 2015; Alonso et al., 2016; Sheldon, 2016; Martins et al., 2018). There is also a consensus that the toxicity and citotoxicity of hydrophilic DESs depend

\section{Previous work:}

"Classical" Ullmann amine synthesis
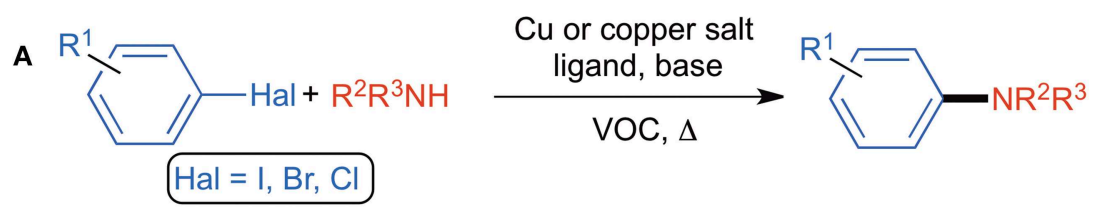

Vaccaro et al. (2018)

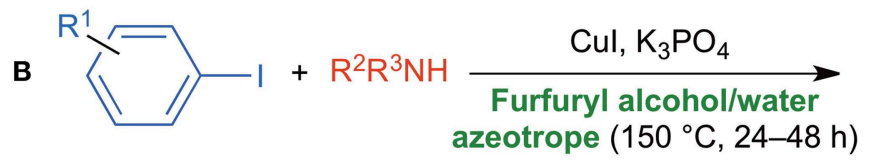

This work:

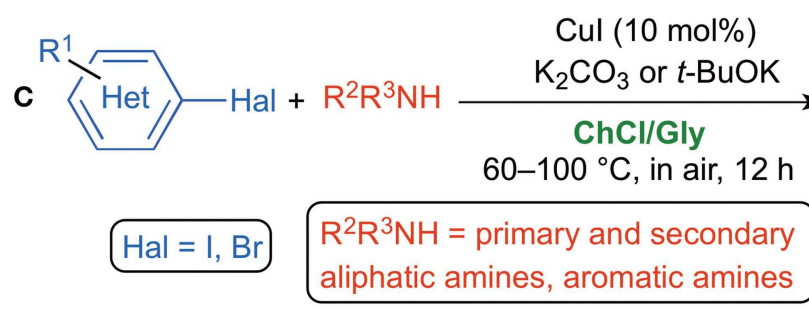

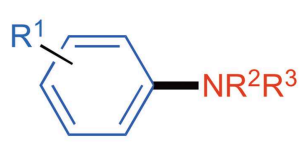

27 examples up to $90 \%$ yield

SCHEME 1 | (A) "Classical" Ullmann amine synthesis; (B) bio-based Ullmann amine synthesis; (C) DES-based Ullmann amine synthesis. 
on their components (Hayyan et al., 2013, 2015, 2016). Those based on naturally occurring and biomass-produced compounds [e.g., amino acids, sugars, vitamins, choline chloride $(\mathrm{ChCl})$ ] benefit of tunable physicochemical properties, biodegradability, easy preparation, non-toxicity and low cost (Hayyan et al., 2013). To date, $N$-arylation of amines in DESs has only been performed employing an engineered copper nanoparticle modified carboxamide-functionalized magnetic graphene oxide as an efficient nanocatalyst. The latter was recovered and reused, jointly with the eutectic mixture, for five consecutive runs (Shaabani and Afshari, 2018). $\mathrm{N}$-arylantraquinone derivatives have also been successfully synthesized, under metal-free conditions and in the presence of choline hydroxide as an environmentally benign and recyclable catalyst, however, via an $\mathrm{S}_{\mathrm{N}} \mathrm{Ar}$ mechanistic pathway (Pant et al., 2018). Building on recent breakthroughs made by our research group (Mallardo et al., 2014; Sassone et al., 2015; Cicco et al., 2016, 2017, 2018; Mancuso et al., 2016; Dilauro et al., 2017, 2018, 2019; Messa et al., 2018; Ghinato et al., 2019) and others (Vidal et al., 2014, 2016; Sánchez-Condado et al., 2019) on the effectiveness of using non-conventional reaction media (e.g., DESs and water) for promoting metal-catalyzed and organolithium/Grignard reagent-mediated deprotonation and nucleophilic addition reactions to unsaturated organic substrates under aerobic conditions, here we report the CuI-catalyzed UAS in DESs. The following features of the proposed protocol are noteworthy: (a) the transformation takes place smoothly in a $\mathrm{ChCl} /$ glycerol (Gly) eutectic mixture under air and moderate heating $\left(60-100^{\circ} \mathrm{C}\right)$, (b) commercially available (hetero)aryl bromides and iodides are employed, (c) the reaction proceeds with no additional ligands and with a broad substrate scope, (d) the expected adducts are isolated in yields up to $98 \%$, and (e) the catalyst, the DES and the base were easily and successfully recycled up to six times (Scheme 1C).

\section{RESULTS AND DISCUSSION}

As DESs are known to be used not only as solvents, but also as catalytic active species, batch reactions to assess the ability of DESs to promote the UAS with no additional ligands were carried out. We initially investigated as a model reaction the coupling between bromobenzene 1a $(0.5 \mathrm{mmol})$ and $N$, $\mathrm{N}$-dimethylethylenediamine $\mathbf{2 a}$ (1 equiv) in different eutectic mixtures for the preparation of adduct 3aa using CuI (10 mol\%) as a catalyst and $\mathrm{K}_{2} \mathrm{CO}_{3}$ as a base (Table 1). Since the beginning, encouraging results were achieved using DESs as reaction media for this kind of reaction. The mixture $\mathrm{ChCl} / \mathrm{Gly}\left(1: 2 \mathrm{~mol} \mathrm{~mol}^{-1}\right)$ was the best among those tested as it delivered adduct $3 \mathrm{aa}$ in $98 \%$ yield after $12 \mathrm{~h}$ heating and stirring at $60^{\circ} \mathrm{C}$ in air (entries 1-4, Table 1). The reaction is characterized by a slow kinetics delivering adduct 3aa in a lower yield at shorter reaction time than $12 \mathrm{~h}$ (Figure 1). On the other hand, no coupling was observed by running the reaction at room temperature, in the absence of the base or CuI, whereas the yield of 3aa dropped to $20-60 \%$ with a catalyst loading inbetween 5-7 mol\% (entries 5-9, Table 1).
TABLE 1 | Optimization of Ullmann amine coupling reaction between bromobenzene $\mathbf{1 a}$ and $\mathrm{N}, \mathrm{N}$-dimethylethylenediamine $\mathbf{2} \mathbf{a}$ to give adduct $\mathbf{3} \mathbf{a} \mathbf{a}^{\mathrm{a}}$.

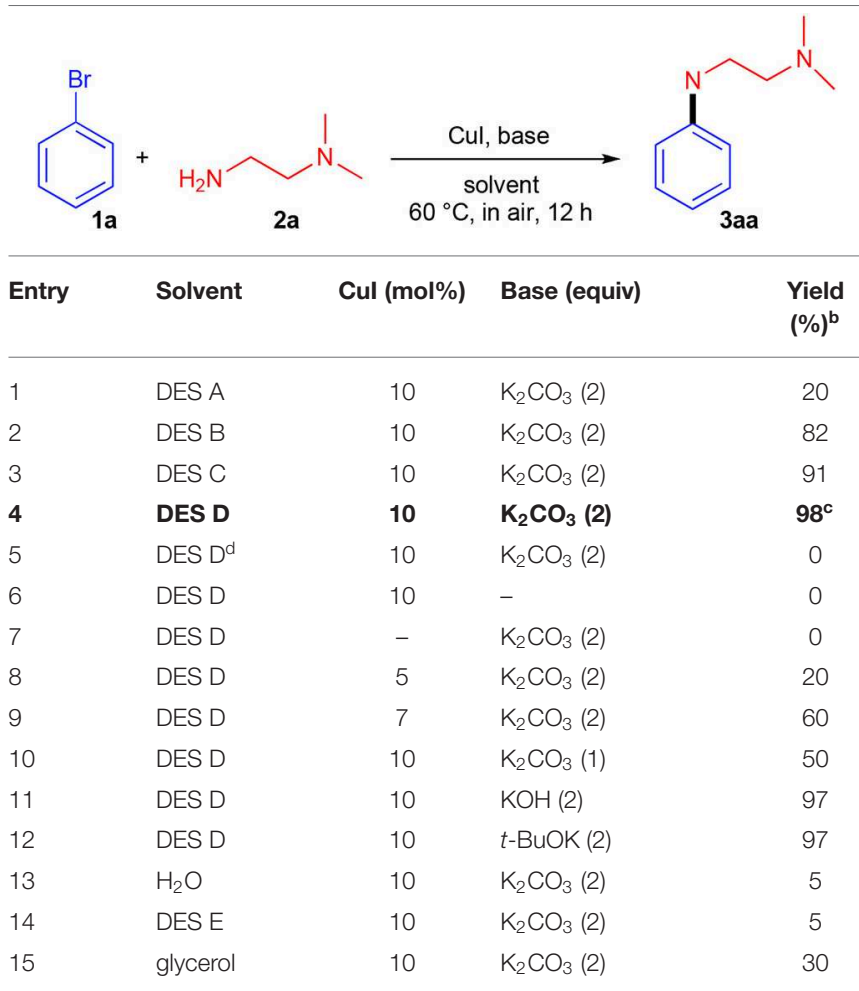

${ }^{a}$ Reaction conditions: bromobenzene $(0.5 \mathrm{mmol}), \mathrm{N}, \mathrm{N}$-dimethylethylenediamine $(1.0$ equiv), Cul, and base were suspended in $1.0 \mathrm{~g} D E S$ or in $1.0 \mathrm{~mL}$ water or glycerol and stirred vigorously at $60^{\circ} \mathrm{C}$ for $12 \mathrm{~h}$. DES A, L-proline/L-lactic acid $\left(1: 2 \mathrm{~mol} \mathrm{~mol}^{-1}\right)$; DES B, choline chloride/urea (1:2 mol mol-1); DES C, L-proline/glycerol $\left(2: 5 \mathrm{~mol} \mathrm{~mol}^{-1}\right) ; D E S$ D, choline chloride/glycerol (1:2 $\left.\mathrm{mol} \mathrm{mol}^{-1}\right)$; DES E, choline chloride/water $\left(1: 2 \mathrm{~mol} \mathrm{~mol}^{-1}\right)$. ${ }^{b}$ Calculated via ${ }^{1} \mathrm{H}$ NMR analysis of the crude reaction mixture using an internal standard technique (NMR internal standard: $\mathrm{CH}_{2} \mathrm{Br}_{2}$ ).

${ }^{c}$ Yield of isolated product.

${ }^{d}$ Room temperature.

A screening of bases also revealed that $\mathrm{K}_{2} \mathrm{CO}_{3}, \mathrm{KOH}$ and $t$ $\mathrm{BuOK}$ were equally effective when used in 2 equiv (entries 10-12, Table 1). Unsatisfactory outcomes in terms of reaction yield (5-30\%) were instead obtained when using water, the $\mathrm{ChCl} / \mathrm{H}_{2} \mathrm{O}\left(1: 2 \mathrm{~mol} \mathrm{~mol}{ }^{-1}\right)$ eutectic mixture or Gly as the solvent (entries 13-15, Table 1). As has been discussed by Ma in an Account (Ma and Cai, 2008), UAS is generally carried out in the presence of bidentate ligands (among which amino acids) which might make $\mathrm{Cu}(\mathrm{I})$ species more reactive toward the oxidative addition reaction or stabilize the oxidative addition intermediates, thereby boosting the coupling process. The fact that the above-described UAS takes place smoothly in DESs in the absence of additional ligands points toward a somewhat stabilizing effect exerted by certain eutectic mixtures on the copper salt, eventually resulting in an improved catalytic performance (Scheme 2).

Adopting the optimized conditions described in Table 1, entry 4 , we then explored the scope of this cross-coupling reaction with a variety of aliphatic primary and secondary amines $(\mathbf{2} \mathbf{a}-\mathbf{g})$ and (hetero)aryl halides (1a-1) (Scheme 3). With regard to diamines $\mathbf{2 a}, \mathbf{b}$ and primary amines $\mathbf{2 c - e}$ relatively high yields (53-98\%) of 


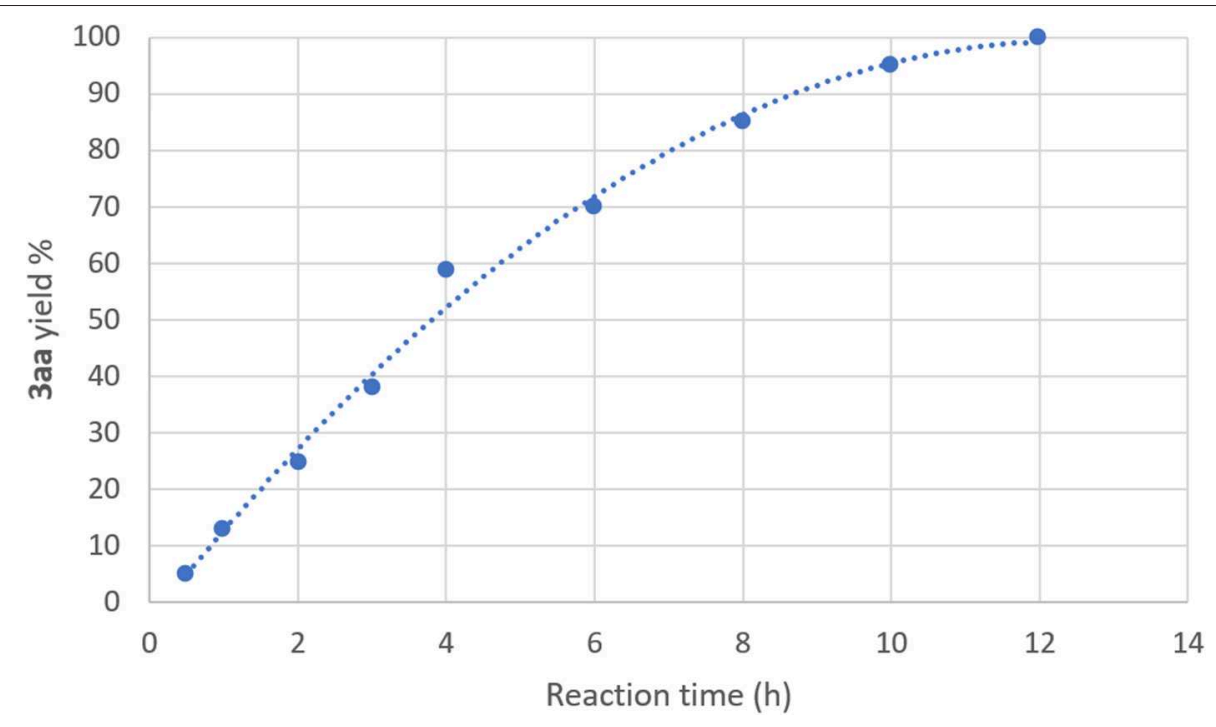

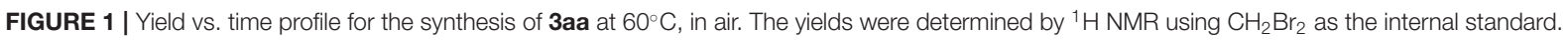

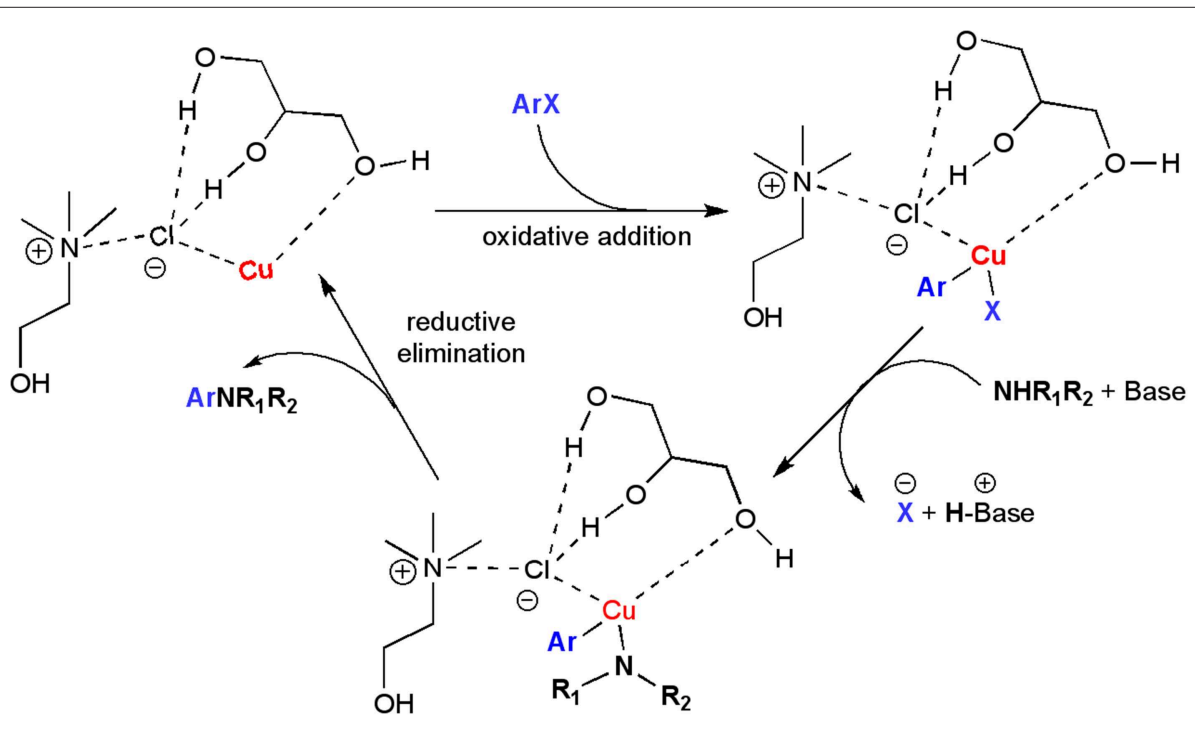

SCHEME 2 | Possible catalytic cycle for the UAS promoted by DES.

the desired coupled products (3ab-3ak, 3bc, 3bk, $3 \mathbf{c a}, 3 \mathbf{c k}, 3 \mathbf{d a}$, $3 \mathrm{eb}, 3 \mathrm{ek}$, and $3 \mathrm{el}$ ) were obtained not only in the reaction with aryl iodides and bromides bearing electron-donating (hydroxy, methoxy) and electron-withdrawing (ester, nitro, cyano, keto) groups at the ortho-, meta-, and para-positions, but also with brominated heterocycles, such as 2- and 3-bromopyridine and 4bromoisoquinoline. Interestingly, both aliphatic and heterocyclic secondary amines $\mathbf{2} \mathbf{f}, \mathbf{g}$ proved to be competent coupling partners as well, and afforded (hetero)aryl derivatives $\mathbf{3 f k}$, 3ga, and $\mathbf{3 g k}$ in $80-90 \%$ yield after simply heating the reaction mixture from 60 to $80^{\circ} \mathrm{C}$.

To further explore the utility of this new protocol, we also investigated the synthesis of diarylamines, which are described in the literature as important molecules due to their antioxidant capacity (Pinto-Basto et al., 2009). Preliminary experiments disclosed that, under the above experimental conditions, aniline (4a) hardly coupled with bromobenzene (1a) as the desired adduct $\mathbf{5}$ aa was delivered in $5 \%$ yield only. Even by increasing the temperature to $100^{\circ} \mathrm{C}$ or by replacing $\mathrm{K}_{2} \mathrm{CO}_{3}$ with the stronger $\mathrm{KOH}$ ( 3 equiv) the 5 aa yield was not higher than $25 \%$ (Scheme 4). To our delight, when the UAS was conducted in the presence of 3 equiv of $t$ - $\mathrm{BuOK}$ at $100^{\circ} \mathrm{C}$, adduct $5 \mathbf{a a}$ now formed in $86 \%$ yield. This percentage could be improved up to $98 \%$ if iodobenzene (1n) was alternatively used as the starting aryl halide. Cross-couplings of $\mathbf{4 a}$ and aniline derivatives $\mathbf{4 b}$, $\mathbf{c}$ with $\mathbf{1 n}$ and assorted aryl iodides and bromides 


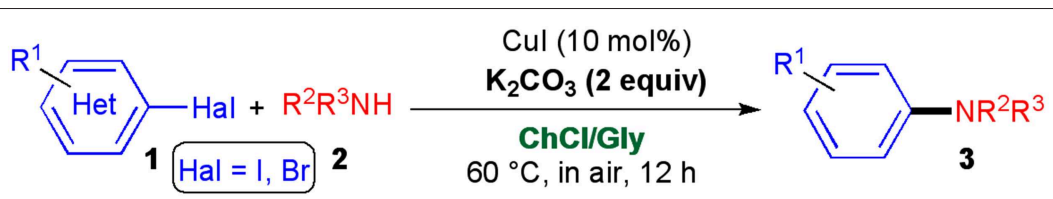

1a: $\mathrm{C}_{6} \mathrm{H}_{5} \mathrm{Br}$; 1b: 4-MeOC $6 \mathrm{H}_{4} \mathrm{Br}$; 1c: 3-MeOC $\mathrm{H}_{4}$; 1d: 2-MeOC $\mathrm{H}_{4} \mathrm{Br}$; 1e: 4-NO $\mathrm{NO}_{6} \mathrm{H}_{4} \mathrm{Br}$; 1f: 4-CNC ${ }_{6} \mathrm{H}_{4} \mathrm{Br}$; 1g: 4-EtOC(O)C ${ }_{6} \mathrm{H}_{4} \mathrm{Br}$; 1h: 3-OHC${ }_{6} \mathrm{H}_{4} \mathrm{Br}$; 1i: 4-Br-isoquinoline; 1j: 3-Brpyridine; 1k: 2-Br-pyridine; 1l: 2-acetyl ${ }_{6} \mathrm{H}_{4} \mathrm{Br}$
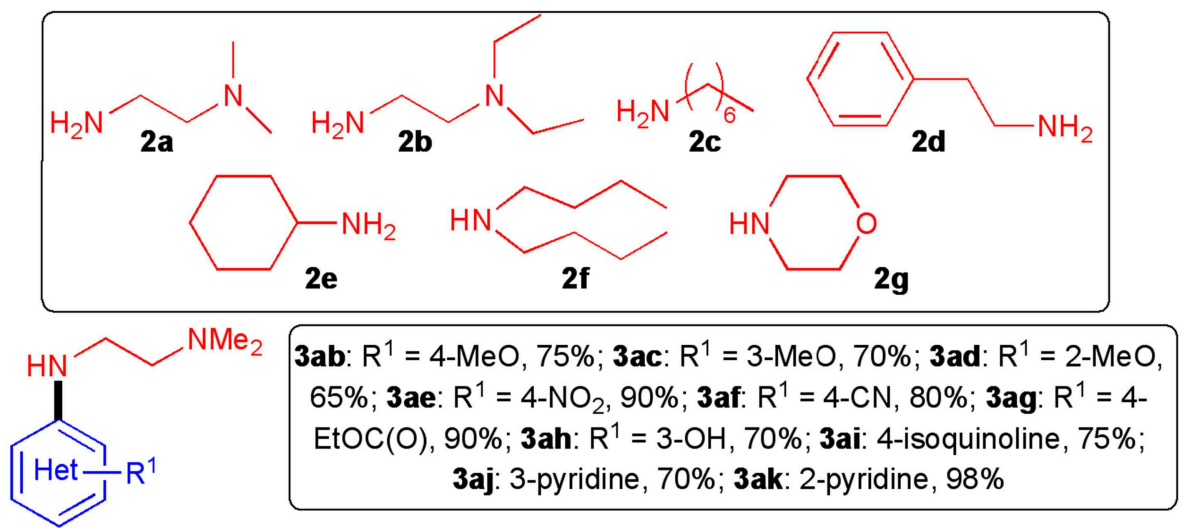<smiles>CCNCCNc1cccc(OC)c1</smiles>

3bc: $70 \%$<smiles>CCNCCNc1ccccn1</smiles>

3bk: $98 \%$<smiles>CC(C)Nc1ccccc1</smiles>

3ca: $97 \%$<smiles>CCCNc1ccccn1</smiles>

3ck: $98 \%$<smiles>c1ccc(CCNc2ccccc2)cc1</smiles>

3da: $85 \%$<smiles>c1ccc(NC2CCCCC2)cc1</smiles>

3eb: $70 \%$<smiles>c1ccc(N2CCOCC2)cc1</smiles>

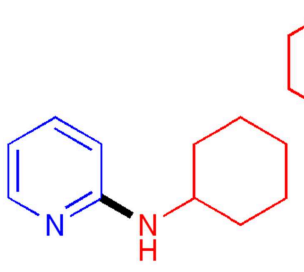

3ek: 53\%<smiles>CC(=O)c1ccccc1NC1CCCCC1</smiles>

3el: $60 \%$<smiles>CCN(c1ccccn1)C(C)C</smiles>

SCHEME 3 | Synthesis of functionalized secondary and tertiary amines $\mathbf{3}$ by copper-catalyzed cross-coupling reactions of (hetero)aryl halides $\mathbf{1}$ with amines $\mathbf{2}$. The yields reported are for products isolated and purified by column chromatography.

substituted with a methoxy (1c, 1o), a hydroxy (1p), and a cyano group (1q) proceeded uneventfully as well in the presence of $t$-BuOK, at $100^{\circ} \mathrm{C}$ in air, leading to functionalised diarylamines 5ac, 5ao, 5ap, 5aq, 5bn, 5bo, and $5 \mathrm{cn}$ in $60-$ 97\% yield (Scheme 4). Of note, under the above conditions, the coupling between $\mathbf{2 a}$ and aryl chlorides, such as chlorobenzene and 3-chloropyridine proved to be ineffective even after $24 \mathrm{~h}$ reaction time.

The catalyst, the DES and the base could be recycled easily. The amination reaction of bromobenzene 1a with diamine 2a was chosen as a model reaction as it provided almost quantitative yield (98\%) of the corresponding adduct 3aa (entry 
<smiles>[R1]c1cccc([AlH2])c1</smiles>

1<smiles>[R]c1ccccc1N</smiles>

4

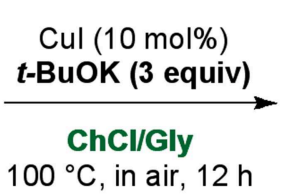

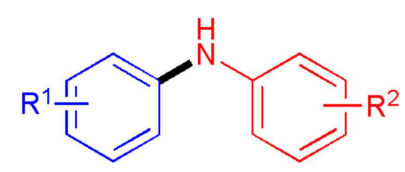

5

1a: $\mathrm{C}_{6} \mathrm{H}_{5} \mathrm{Br}$; 1c: 3- $\mathrm{MeOC}_{6} \mathrm{H}_{4} \mathrm{l}$; 1n: $\mathrm{C}_{6} \mathrm{H}_{5} \mathrm{l}$; 1o: 4- $\mathrm{MeOC}_{6} \mathrm{H}_{4} \mathrm{l}$; 1p: $3-\mathrm{OHC}_{6} \mathrm{H}_{4}$; 1 1q: 3-CN-4- $\mathrm{MeC}_{6} \mathrm{H}_{3} \mathrm{Br}$
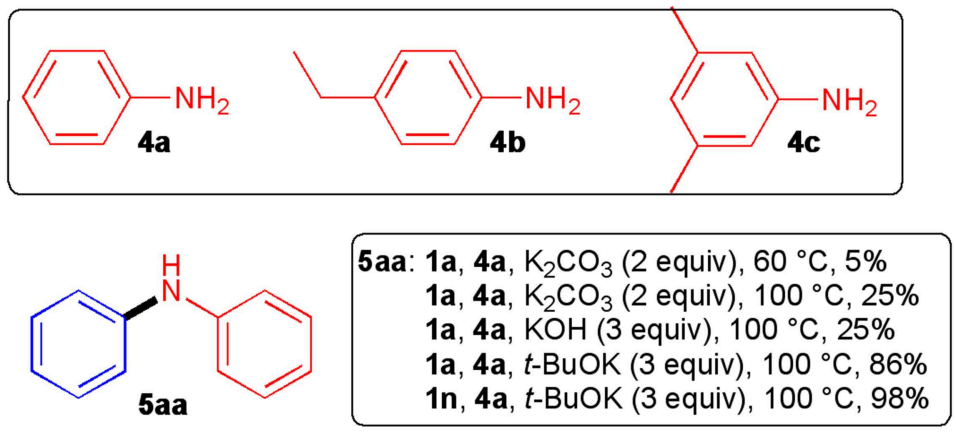<smiles>COc1cccc(Nc2ccccc2)c1</smiles><smiles>COc1ccc(Nc2ccccc2)cc1</smiles><smiles></smiles><smiles>Cc1ccc(Nc2ccccc2)cc1C#N</smiles>

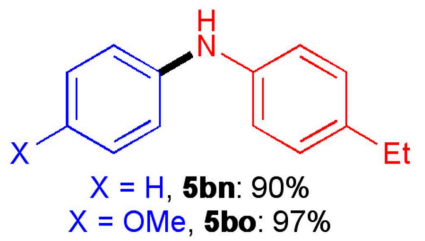<smiles>Cc1cc(C)cc(Nc2ccccc2)c1</smiles>

SCHEME 4 | Synthesis of diarylamines 5 by copper-catalyzed cross-coupling reactions of aryl halides $\mathbf{1}$ with arylamines $\mathbf{4}$. The yields reported are for products isolated and purified by column chromatography.

4, Table 1). Once the stirring was stopped after $12 \mathrm{~h}$ reaction time, in-flask extraction with cyclopentyl methyl ether (CPME) (Watanabe et al., 2007; Azzena et al., 2019) (1 mL), which is an environmentally responsible solvent, afforded product 3 aa $(98 \%$ yield, determined by ${ }^{1} \mathrm{H}$ NMR, Figure 2, number of cycles = 1 ), but leaving the active copper species in the eutectic mixture. Then, upon the addition of new, fresh reagents, the catalyst, the DES and the base could be successfully re-used for further reaction runs. As shown in Figure 2, the catalyst remained active over 6 cycles, with a decrease in the final yield of 3aa of up to 2\%: $98 \%$ (second run), 97\% (third run), 97\% (fourth run), $97 \%$ (fifth run), and $96 \%$ (sixth run). In order to compare the reaction rates observed for each run, we re-investigated the above catalytic cycle at a lower conversion. Thus, each run was stopped after $3 \mathrm{~h}$ reaction time and the corresponding yield of $3 \mathrm{aa}$ calculated: $38 \%$ (first, second, and third run), 36\% (fourth run), 38\% (fifth and sixth run), and $36 \%$ (seventh run). Overall, these results confirmed the excellent performance of the copper species as catalyst in the employed eutectic mixture. Finally, to evaluate the sustainability of the proposed protocol and to provide metrics for the "green" aspects herein discussed, we calculated the E-factor (kg of waste/kg of product) (Sheldon, 2007; Roschangar et al., 2015) for the recycling process, obtaining a value as low as 13.8 (10 mol\% CuI, 6 cycles, see Supplementary Material for details).

\section{CONCLUSION}

In summary, a practical and efficient protocol has been developed for performing CuI-catalyzed Ullmann amine synthesis using a biodegradable $\mathrm{ChCl}$-based eutectic mixture as an environmentally responsible reaction medium in place of toxic and hazardous VOCs. Valuable aspects of the proposed protocol are: (a) cross-coupling reactions between (hetero)aryl bromides and iodides and a variety of aromatic and aliphatic primary and secondary amines run under mild conditions $\left(60-100^{\circ} \mathrm{C}\right)$ in air, with $\mathrm{K}_{2} \mathrm{CO}_{3}$ or $t$-BuOK as the base, and a catalyst loading of $\mathrm{CuI}$ of up to $10 \mathrm{~mol} \%$; (b) the absence of additional ligands; (c) good reaction efficiency starting from both electron-deficient and electron-rich (hetero)aryl halides; (d) 


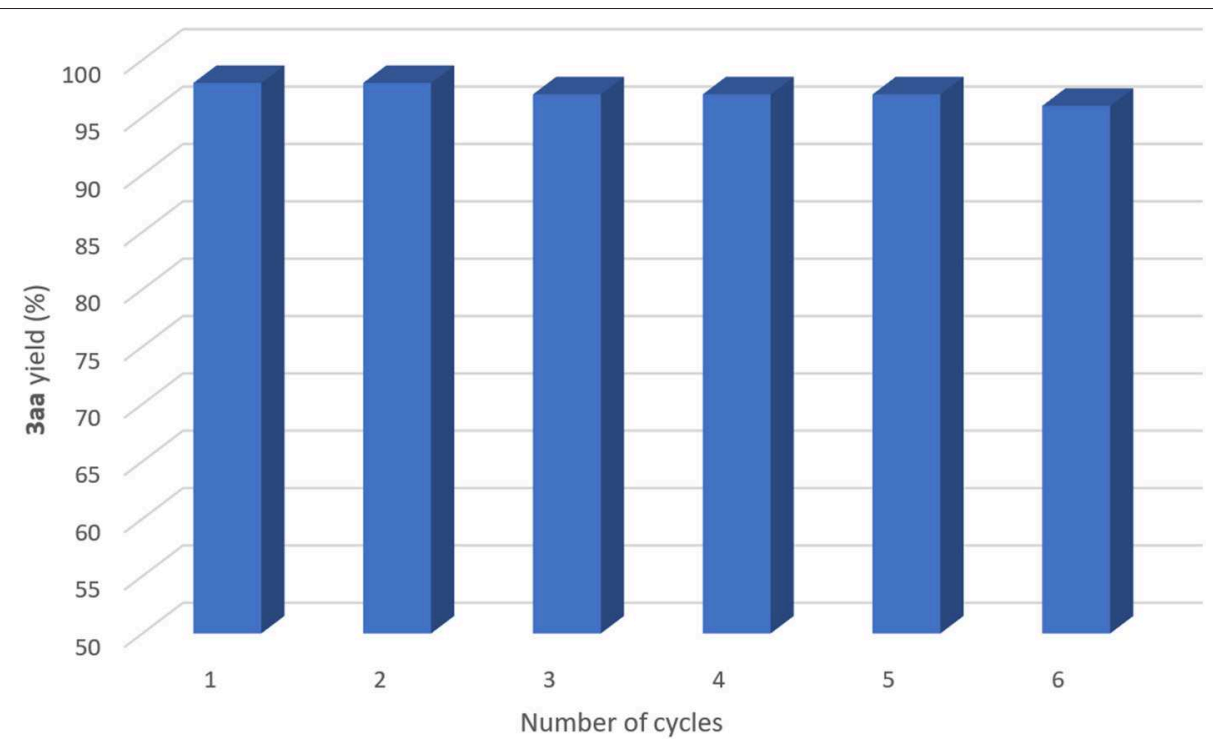

FIGURE 2 | Recycling of Cul, DES, and $\mathrm{K}_{2} \mathrm{CO}_{3}$ in the coupling reaction between bromobenzene (1a) and $\mathrm{N}, \mathrm{N}$-dimethylethylenediamine (2a) with 10 mol\% catalyst. The yields were determined by ${ }^{1} \mathrm{H}$ NMR using $\mathrm{CH}_{2} \mathrm{Br}_{2}$ as the internal standard.

broad substrate scope and tolerance of several functional groups with the expected adducts isolated in up to $98 \%$ yield; (e) effective recycling of the DES, the catalyst and the base. Efforts toward targeting and producing medicinally relevant drug compounds on a larger scale are underway and will be reported in due course.

\section{MATERIALS AND METHODS}

\section{General Methods}

${ }^{1} \mathrm{H}$ NMR and ${ }^{13} \mathrm{C}$ NMR spectra were recorded on a Bruker $600 \mathrm{MHz}$ spectrometer and chemical shifts are reported in parts per million ( $\delta$ ). FT-IR spectra were recorded on a Perkin-Elmer 681 spectrometer. GC analyses were performed on a HP 6890 model, Series II by using a HP1 column (methyl siloxane; $30 \mathrm{~m} \times 0.32 \mathrm{~mm} \times 0.25 \mu \mathrm{m}$ film thickness). Analytical thin-layer chromatography (TLC) was carried out on pre-coated $0.25 \mathrm{~mm}$ thick plates of Kieselgel 60 F254; visualization was accomplished by UV light $(254 \mathrm{~nm})$ or by spraying a solution of $5 \%(\mathrm{w} / \mathrm{v})$ ammonium molybdate and $0.2 \%(\mathrm{w} / \mathrm{v})$ cerium(III) sulfate in $100 \mathrm{~mL} 17.6 \%(\mathrm{w} / \mathrm{v})$ aq. sulfuric acid and heating to $473 \mathrm{~K}$ until blue spots appeared. Chromatography was conducted by using silica gel 60 with a particle size distribution $40-63 \mu \mathrm{m}$ and $230-$ 400 ASTM. GC-MS analyses were performed on HP 5995C model. Cyclopentyl methyl ether (CPME) was used as the solvent in the work-up procedures. High-resolution mass spectrometry (HRMS) analyses were performed using a Bruker microTOF QII mass spectrometer equipped with an electrospray ion source (ESI). CP-OES analysis was performed on a Spectro Arcos FHE 12 (Ametek) spectrometer. Reagents and solvents, unless otherwise specified, were purchased from Sigma-Aldrich (Sigma-Aldrich, St. Louis, MO, USA) and used without any further purification. (Hetero)aryl halides and amines used for the synthesis of compounds $\mathbf{3}$ and $\mathbf{5}$ are commercially available. Copies of ${ }^{1} \mathrm{H}$ and ${ }^{13} \mathrm{C}$ NMR spectra are available in the Supplementary Material. Deep Eutectic Solvents [DES A: L-proline/L-lactic acid (1:2 $\left.\mathrm{mol} \mathrm{mol}^{-1}\right)$; DES B: choline chloride/urea (1:2 $\left.\mathrm{mol} \mathrm{mol}^{-1}\right)$; DES C: L-proline/glycerol (2:5 mol mol $\left.{ }^{-1}\right)$; DES D: choline chloride/glycerol $(1: 2 \mathrm{~mol}$ $\left.\mathrm{mol}^{-1}\right)$; DES E: choline chloride/water $\left.\left(1: 2 \mathrm{~mol} \mathrm{~mol}^{-1}\right)\right]$ were prepared by heating under stirring at $60-80^{\circ} \mathrm{C}$ for $10-30 \mathrm{~min}$ the corresponding individual components until a clear solution was obtained.

\section{Typical Procedure for the Ullmann Cross-Coupling Reaction With Aliphatic Amines: Synthesis of $N^{1}, N^{1}$-dimethyl- $N^{2}$-phenylethane-1,2-diamine (3aa)}

$\mathrm{CuI}$ (10 mol\%, $0.05 \mathrm{mmol}, 10 \mathrm{mg}$ ), bromobenzene (1a, 1 equiv, $0.5 \mathrm{mmol}, 79 \mathrm{mg}, 53 \mu \mathrm{L}), N, N$-dimethylethylenediamine (2a, 1 equiv, $0.5 \mathrm{mmol}, 44 \mathrm{mg}, 55 \mu \mathrm{L})$ and the base $\left(\mathrm{K}_{2} \mathrm{CO}_{3}, 2\right.$ equiv, $1 \mathrm{mmol}, 138 \mathrm{mg}$ ) were suspended in $1 \mathrm{~g}$ DES (Gly/ChCl, 2:1 mol $\mathrm{mol}^{-1}$ ), under air, in a vial with a Teflon screw tap under vigorous stirring at $60^{\circ} \mathrm{C}$. The reaction mixture was monitored by GC. After $12 \mathrm{~h}$, the mixture was cooled to room temperature and $1 \mathrm{~mL}$ of $\mathrm{H}_{2} \mathrm{O}$ was added. Then, the mixture was extracted with CPME $(3 \times 1 \mathrm{~mL})$ and the organic phase was dried over anhydrous $\mathrm{Na}_{2} \mathrm{SO}_{4}$ and filtered over a celite pad. Evaporation of the solvent under reduced pressure afforded the crude that was purified by flash-chromatography on silica gel (hexane/AcOEt $8: 2$ ) to provide the desired product 3 aa in $98 \%$ yield $(80 \mathrm{mg})$.

\section{$N^{1}, N^{1}$-Dimethyl- $N^{2}$-phenylethane-1,2-diamine (3aa)}

Colorless oil, yield $98 \%$.

${ }^{1} \mathrm{H}$ NMR (600 MHz, $\left.\mathrm{CDCl}_{3}\right) \delta 2.27(\mathrm{~s}, 6 \mathrm{H}), 2.56-2.58(\mathrm{~m}$, $2 \mathrm{H}), 3.16-3.17(\mathrm{~m}, 2 \mathrm{H}), 6.65(\mathrm{~d}, J=7.2 \mathrm{~Hz}, 2 \mathrm{H}), 6.72(\mathrm{t}, J=$ $7.2 \mathrm{~Hz}, 1 \mathrm{H}), 7.20(\mathrm{~d}, J=7.2 \mathrm{~Hz}, 2 \mathrm{H})$.

${ }^{13} \mathrm{C} \mathrm{NMR}\left(150 \mathrm{MHz}, \mathrm{CDCl}_{3}\right) \delta 42.2,45.2,58.1,112.9,117.2$, 129.2, 148.6. 
FT-IR (film, $\mathrm{cm}^{-1}$ ): 3379, 3051, 2920, 2821, 1728, 1603, 1505, 1463, 1258, 1041, 748, 700 .

GC/MS (70 eV) m/z (\%): $164\left(\mathrm{M}^{+}, 15\right), 106(10), 77$ (9), 58 (100), 42 (6).

HRMS (ESI) $m / z$ calcd for $\left[\mathrm{C}_{10} \mathrm{H}_{16} \mathrm{~N}_{2}+\mathrm{H}\right]^{+}:$165.1386; found: 165.1393 .

\section{$N^{1}$-(4-Methoxyphenyl)- $N^{2}, N^{2}$-dimethylethane-1,2- diamine (3ab)}

Colorless oil, yield 75\%.

${ }^{1} \mathrm{H} \mathrm{NMR}\left(600 \mathrm{MHz}, \mathrm{CDCl}_{3}\right) \delta 2.26(\mathrm{~s}, 6 \mathrm{H}), 2.56(\mathrm{t}, J=6.0 \mathrm{~Hz}$, $2 \mathrm{H}), 3.12(\mathrm{t}, J=6.0 \mathrm{~Hz}, 2 \mathrm{H}), 3.76(\mathrm{~s}, 3 \mathrm{H}), 6.62(\mathrm{~d}, J=7.6 \mathrm{~Hz}$, $2 \mathrm{H}), 6.80(\mathrm{~d}, J=7.6 \mathrm{~Hz}, 2 \mathrm{H})$.

${ }^{13} \mathrm{C} \mathrm{NMR}\left(150 \mathrm{MHz}, \mathrm{CDCl}_{3}\right) \delta 42.2,45.3,55.9,58.2,114.2$, $114.9,143.0,152.1$.

FT-IR (film, $\mathrm{cm}^{-1}$ ): 3368, 2942, 1619, 1514, 1464, 1288, 1243, $1179,1039,959,820$.

GC/MS (70 eV) m/z (\%): $194\left(\mathrm{M}^{+}, 35\right), 136$ (42), 135 (18), 121 (61), 108 (59), 77 (5), 59 (9), 58 (100), 42 (6).

HRMS (ESI) $m / z$ calcd for $\left[\mathrm{C}_{11} \mathrm{H}_{18} \mathrm{~N}_{2} \mathrm{O}+\mathrm{H}\right]^{+}:$195.1492; found: 195.1495 .

\section{$N^{1}$-(3-Methoxyphenyl)- $N^{2}, N^{2}$-dimethylethane-1,2-} diamine (3ac)

Colorless oil, yield $70 \%$.

${ }^{1} \mathrm{H} \mathrm{NMR}\left(600 \mathrm{MHz}, \mathrm{CDCl}_{3}\right) \delta 2.24(\mathrm{~s}, 6 \mathrm{H}), 2.54(\mathrm{t}, J=5.9 \mathrm{~Hz}$, $2 \mathrm{H}), 3.12(\mathrm{t}, J=5.9 \mathrm{~Hz}, 2 \mathrm{H}), 3.75(\mathrm{~s}, 3 \mathrm{H}), 6.18(\mathrm{~s}, 1 \mathrm{H}), 6.23-6.26$ $(\mathrm{m}, 2 \mathrm{H}), 7.06(\mathrm{t}, J=8.0 \mathrm{~Hz}, 1 \mathrm{H})$.

${ }^{13} \mathrm{C}$ NMR $\left(150 \mathrm{MHz}, \mathrm{CDCl}_{3}\right) \delta 41.1,45.1,55.0,58.0,98.7$, 102.4, 106.0, 129.9, 149.9, 160.8 .

FT-IR (film, $\mathrm{cm}^{-1}$ ): 3400, 2924, 2852, 1736, 1602, 1516, 1457, 1378, 1346, 1252, 1179, 1128, 1026, 916, 737.

GC/MS (70 eV) m/z (\%): $194\left(\mathrm{M}^{+}, 30\right), 136(36), 135$ (16), 92 (5), 59 (9), 58 (100), 42 (6).

HRMS (ESI) $m / z$ calcd for $\left[\mathrm{C}_{11} \mathrm{H}_{18} \mathrm{~N}_{2} \mathrm{O}+\mathrm{H}\right]^{+}:$195.1492; found: 195.1493 .

\section{$N^{1}$-(2-Methoxyphenyl)- $\mathrm{N}^{2}, \mathrm{~N}^{2}$-dimethylethane-1,2- diamine (3ad)}

Colorless oil, yield $65 \%$.

${ }^{1} \mathrm{H} \mathrm{NMR}\left(600 \mathrm{MHz}, \mathrm{CDCl}_{3}\right) \delta 2.29(\mathrm{~s}, 6 \mathrm{H}), 2.61(\mathrm{t}, J=6.2 \mathrm{~Hz}$, $2 \mathrm{H}), 3.22(\mathrm{t}, J=6.2 \mathrm{~Hz}, 2 \mathrm{H}), 3.86(\mathrm{~s}, 3 \mathrm{H}), 6.61(\mathrm{~d}, J=7.6 \mathrm{~Hz}$, $1 \mathrm{H}), 6.67(\mathrm{t}, J=7.6 \mathrm{~Hz}, 1 \mathrm{H}), 6.77(\mathrm{~d}, J=7.6 \mathrm{~Hz}, 1 \mathrm{H}), 6.88(\mathrm{t}, J$ $=7.6 \mathrm{~Hz}, 1 \mathrm{H})$.

${ }^{13} \mathrm{C}$ NMR $\left(150 \mathrm{MHz}, \mathrm{CDCl}_{3}\right) \delta 41.2,45.3,55.4,58.3,109.4$, $109.8,116.4,121.2,138.4,147.1$.

FT-IR (film, $\mathrm{cm}^{-1}$ ): 3401, 2926, 2819, 2768, 1602, 1519, 1238, $1130,733$.

GC/MS (70 eV) m/z (\%): $194\left(\mathrm{M}^{+}, 26\right), 136(28), 121(16), 120$ (23), 77 (6), 65 (6), 58 (100), 42(8). HRMS (ESI) $\mathrm{m} / z$ calcd for $\left[\mathrm{C}_{11} \mathrm{H}_{18} \mathrm{~N}_{2} \mathrm{O}+\mathrm{H}\right]^{+}:$195.1492; found: 195.1493 .

\section{$N^{1}, N^{1}$-Dimethyl- $N^{2}$-(4-nitrophenyl)ethane-1,2-} diamine (3ae)

Yellow oil, yield 90\%.
${ }^{1} \mathrm{H}$ NMR $\left(600 \mathrm{MHz}, \mathrm{CDCl}_{3}\right) \delta 2.26(\mathrm{~s}, 6 \mathrm{H}), 2.54-2.57(\mathrm{~m}$, $2 \mathrm{H}), 3.15-3.16(\mathrm{~m}, 2 \mathrm{H}), 4.94$ (br. s, $1 \mathrm{H}), 6.56(\mathrm{~d}, J=7.6 \mathrm{~Hz}$, $2 \mathrm{H}), 7.42(\mathrm{~d}, J=7.6 \mathrm{~Hz}, 2 \mathrm{H})$.

${ }^{13} \mathrm{C} \mathrm{NMR}\left(150 \mathrm{MHz}, \mathrm{CDCl}_{3}\right) \delta 40.0,44.8,57.1,111.1,130.0$, 137.9, 153.3 .

FT-IR (film, $\mathrm{cm}^{-1}$ ): 3369, 2920, 1601, 1466, 1307, 1111, 838

GC/MS (70 eV) m/z (\%): $209\left(\mathrm{M}^{+}, 22\right), 120$ (5), $76(6), 58$ (100), $51(8), 42(7)$.

HRMS (ESI) $m / z$ calcd for $\left[\mathrm{C}_{10} \mathrm{H}_{15} \mathrm{~N}_{3} \mathrm{O}_{2}+\mathrm{H}\right]^{+}:$: 10.1237 ; found: 210.1239 .

\section{4-\{[2-(Dimethylamino)ethyl]amino\}benzonitrile (3af)}

Colorless oil, yield $80 \%$.

${ }^{1} \mathrm{H}$ NMR $\left(600 \mathrm{MHz}, \mathrm{CDCl}_{3}\right) \delta 2.27(\mathrm{~s}, 6 \mathrm{H}), 2.57-2.59(\mathrm{~m}$, $2 \mathrm{H}), 3.15-3.18(\mathrm{~m}, 2 \mathrm{H}), 4.97$ (br. s, $1 \mathrm{H}), 6.56(\mathrm{~d}, J=7.6 \mathrm{~Hz}$, $2 \mathrm{H}), 7.41(\mathrm{~d}, J=7.6 \mathrm{~Hz}, 2 \mathrm{H})$.

${ }^{13} \mathrm{C}$ NMR $\left(150 \mathrm{MHz}, \mathrm{CDCl}_{3}\right) \delta 40.1,45.0,57.3,98.4,112.2$, 120.6, 133.7, 151.5.

FT-IR (film, cm ${ }^{-1}$ ): 3367, 2922, 2855, 2777, 2212, 1721, 1669, 1608, 1526, 1469, 1338, 1173, 1132, 1041, 825, 732.

GC/MS (70 eV) m/z (\%): $189\left(\mathrm{M}^{+}, 6\right), 102(6), 58$ (100), $42(6)$. HRMS (ESI) $m / z$ calcd for $\left[\mathrm{C}_{11} \mathrm{H}_{15} \mathrm{~N}_{3}+\mathrm{H}\right]^{+}:$190.1339; found: 190.1341 .

\section{Ethyl}

\section{4-\{[2-(dimethylamino)ethyl]amino\}benzoate (3ag)}

Yellow oil, yield $90 \%$.

${ }^{1} \mathrm{H} \mathrm{NMR}\left(600 \mathrm{MHz}, \mathrm{CDCl}_{3}\right) \delta 1.11(\mathrm{t}, J=7.0 \mathrm{~Hz}, 3 \mathrm{H}), 2.26(\mathrm{~s}$, $6 \mathrm{H}), 2.53-2.57(\mathrm{~m}, 2 \mathrm{H}), 3.18-3.19(\mathrm{~m}, 2 \mathrm{H}), 4.33-4.41(\mathrm{~m}, 2 \mathrm{H})$, 6.56-6.58 (m, $2 \mathrm{H}), 7.86-7.88(\mathrm{~m}, 2 \mathrm{H})$.

${ }^{13} \mathrm{C} \mathrm{NMR}\left(150 \mathrm{MHz}, \mathrm{CDCl}_{3}\right) \delta 14.5,40.3,45.0,57.6,60.1$, $111.5,118.4,131.5,152.0,167.0$

FT-IR (film, $\mathrm{cm}^{-1}$ ): 3395, 2922, 2852, 1682, 1607, 1464, 1341, 1276, 1110, 1041, 839, 700.

GC/MS (70 eV) m/z (\%): $136\left(\mathrm{M}^{+}, 6\right), 191$ (6), 150 (6), 105 (7), 58 (100).

HRMS (ESI) $m / z$ calcd for $\left[\mathrm{C}_{13} \mathrm{H}_{20} \mathrm{~N}_{2} \mathrm{O}_{2}+\mathrm{H}\right]^{+}:$237.1598; found: 237.1600 .

\section{3-\{[2-(Dimethylamino)ethyl]amino\}phenol (3ah)}

Yellow oil, yield 70\%.

${ }^{1} \mathrm{H}$ NMR $\left(600 \mathrm{MHz}, \mathrm{CDCl}_{3}\right) \delta 2.26(\mathrm{~s}, 6 \mathrm{H}), 2.55-2.57(\mathrm{~m}$, $2 \mathrm{H}), 3.14-3.15(\mathrm{~m}, 2 \mathrm{H}), 6.12(\mathrm{~s}, 1 \mathrm{H}), 6.16(\mathrm{~d}, J=8.0 \mathrm{~Hz}, 1 \mathrm{H})$, $6.23(\mathrm{~d}, J=8.0 \mathrm{~Hz}, 1 \mathrm{H}), 7.02(\mathrm{t}, J=8.0 \mathrm{~Hz}, 1 \mathrm{H})$.

${ }^{13} \mathrm{C}$ NMR $\left(150 \mathrm{MHz}, \mathrm{CDCl}_{3}\right) \delta 41.0,45.1,57.9,99.7,104.4$, 105.8, 134.8, 149.9, 156.7.

FT-IR (film, $\mathrm{cm}^{-1}$ ): 3368, 2928, 1605, 1463, 1261, 1047.

GC/MS (70 eV) m/z (\%): $180\left(\mathrm{M}^{+}, 16\right), 122(10), 65(6), 58$ (100), 42 (7).

HRMS (ESI) $m / z$ calcd for $\left[\mathrm{C}_{10} \mathrm{H}_{16} \mathrm{~N}_{2} \mathrm{O}+\mathrm{H}\right]^{+}: 181.1335$; found: 181.1336 .

\section{$N^{1}$-(Isoquinolin-4-yl)- $N^{2}, N^{2}$-dimethylethane-1,2-} diamine (3ai)

Colorless oil, yield 75\%.

${ }^{1} \mathrm{H}$ NMR $\left(600 \mathrm{MHz}, \mathrm{CDCl}_{3}\right) \delta 2.38(\mathrm{~s}, 6 \mathrm{H}), 2.80-2.81(\mathrm{~m}$, $2 \mathrm{H}), 3.39-3.40$ (m, $2 \mathrm{H}), 5.27$ (br. s, $1 \mathrm{H}), 7.58-7.61(\mathrm{~m}, 1 \mathrm{H})$, 
7.66-7.69 (m, 1H), 7.85-7.86 (m, 2H), 7.91-7.92 (m, 1H), $8.70(\mathrm{~s}, 1 \mathrm{H})$.

${ }^{13} \mathrm{C}$ NMR $\left(150 \mathrm{MHz}, \mathrm{CDCl}_{3}\right) \delta 46.6,47.3,60.3,121.7,124.6$, $126.5,126.9,128.3,128.9,129.8,134.2,140.3$.

FT-IR (film, $\mathrm{cm}^{-1}$ ): 3391, 2918, 1724, 1651, 1583, 1407, 1285, $1121,1040,842,751$.

GC/MS (70 eV) m/z (\%): $215\left(\mathrm{M}^{+}, 15\right), 157(8), 128$ (6), 101 (5), 58 (100), 42 (6).

HRMS (ESI) $m / z$ calcd for $\left[\mathrm{C}_{13} \mathrm{H}_{17} \mathrm{~N}_{3}+\mathrm{H}\right]^{+}:$216.1495; found: 216.1500 .

\section{$N^{1}, N^{1}$-Dimethyl- $N^{2}$-(pyridin-3-yl)ethane-1,2-} diamine (3aj)

Yellow oil, yield $70 \%$.

${ }^{1} \mathrm{H}$ NMR $\left(600 \mathrm{MHz}, \mathrm{CDCl}_{3}\right) \delta 2.27(\mathrm{~s}, 6 \mathrm{H}), 2.57-2.59(\mathrm{~m}$, $2 \mathrm{H}), 3.14-3.15$ (m, $2 \mathrm{H}), 4.37$ (br. s, $1 \mathrm{H}), 6.88-6.90(\mathrm{~m}, 1 \mathrm{H})$, 7.09-7.10 (m, $1 \mathrm{H}), 7.97-7.98(\mathrm{~m}, 1 \mathrm{H}), 8.07$ (s, $1 \mathrm{H})$.

${ }^{13} \mathrm{C} \mathrm{NMR}\left(150 \mathrm{MHz}, \mathrm{CDCl}_{3}\right) \delta 40.6,45.1,57.7,118.6,123.7$, 136.1, 138.6, 144.5.

FT-IR (film, $\mathrm{cm}^{-1}$ ): 3335, 2924, 2855, 1652, 1592, 1470, 1421, 1302, 1248, 1192, 1133, 1042, 874, 797, 709.

GC/MS (70 eV) m/z (\%): $165\left(\mathrm{M}^{+}, 8\right), 107$ (5), 78 (6), 58 (100), 51 (5), 42 (8).

HRMS (ESI) $m / z$ calcd for $\left[\mathrm{C}_{9} \mathrm{H}_{15} \mathrm{~N}_{3}+\mathrm{H}\right]^{+}:$166.1339; found: 166.1339 .

\section{$N^{1}, N^{1}$-Dimethyl- $N^{2}$-(pyridin-2-yl)ethane-1,2- diamine (3ak)}

Yellow oil, yield $98 \%$.

${ }^{1} \mathrm{H}$ NMR $\left(600 \mathrm{MHz}, \mathrm{CDCl}_{3}\right) \delta 2.27(\mathrm{~s}, 6 \mathrm{H}), 2.54-2.56(\mathrm{~m}$, $2 \mathrm{H}), 3.34-3.36(\mathrm{~m}, 2 \mathrm{H}), 5.01$ (br. s, $1 \mathrm{H}), 6.41(\mathrm{~d}, J=7.3 \mathrm{~Hz}$, $1 \mathrm{H}), 6.56(\mathrm{t}, J=7.3 \mathrm{~Hz}, 1 \mathrm{H}), 7.40(\mathrm{t}, J=7.3 \mathrm{~Hz}, 1 \mathrm{H}), 8.09-8.10$ $(\mathrm{m}, 1 \mathrm{H})$.

${ }^{13} \mathrm{C} \mathrm{NMR}\left(150 \mathrm{MHz}, \mathrm{CDCl}_{3}\right) \delta 39.4,45.2,58.0,107.4,112.6$, 137.2, 148.0, 158.8 .

FT-IR (film, $\mathrm{cm}^{-1}$ ): 3325, 2956, 2926, 2855, 1667, 1455, 1152, $769,734$.

GC/MS (70 eV) m/z (\%): $165\left(\mathrm{M}^{+}, 8\right), 107$ (5), 95 (6), 78 (6), 71 (9), 58 (100), 51 (5), 42 (8).

HRMS (ESI) $m / z$ calcd for $\left[\mathrm{C}_{9} \mathrm{H}_{15} \mathrm{~N}_{3}+\mathrm{H}\right]^{+}$: 166.1339; found: 166.1342 .

\section{$N^{1}, N^{1}$-Diethyl- $N^{2}$-(3-methoxyphenyl)ethane-1,2- diamine (3bc)}

Colorless oil, yield $70 \%$.

${ }^{1} \mathrm{H}$ NMR $\left(600 \mathrm{MHz}, \mathrm{CDCl}_{3}\right) \delta 1.03(\mathrm{t}, J=7.0 \mathrm{~Hz}, 6 \mathrm{H}), 2.56$ $(\mathrm{q}, J=7.0 \mathrm{~Hz}, 4 \mathrm{H}), 2.69(\mathrm{t}, J=5.8 \mathrm{~Hz}, 2 \mathrm{H}), 3.13(\mathrm{t}, J=5.8 \mathrm{~Hz}$, $2 \mathrm{H}), 6.19$ (s, $1 \mathrm{H}), 6.25-6.27(\mathrm{~m}, 3 \mathrm{H}), 7.07$ (t, $J=8.0 \mathrm{~Hz}, 1 \mathrm{H})$.

${ }^{13} \mathrm{C} \mathrm{NMR}\left(150 \mathrm{MHz}, \mathrm{CDCl}_{3}\right) \delta 11.7,29.7,46.7,55.1,55.4$, $102.4,106.2,113.8,123.0,149.0,150.1$.

FT-IR (film, $\mathrm{cm}^{-1}$ ): 3380, 2962, 2925, 2852, 1732, 1614, 1585, 1504, 1474, 1425, 1381, 1285, 1243, 1229, 1211, 1161, 1034, 989.

GC/MS (70 eV) m/z (\%): $222\left(\mathrm{M}^{+}, 13\right), 136(9), 92(6), 86$ (100), 58 (6).

HRMS (ESI) $m / z$ calcd for $\left[\mathrm{C}_{13} \mathrm{H}_{22} \mathrm{~N}_{2} \mathrm{O}+\mathrm{H}\right]^{+}:$223.1805; found: 223.1808 .
$N^{1}, N^{1}$-Diethyl- $N^{2}$-(pyridin-2-yl)ethane-1,2-

diamine (3bk)

Colorless oil, yield $97 \%$.

${ }^{1} \mathrm{H}$ NMR $\left(600 \mathrm{MHz}, \mathrm{CDCl}_{3}\right) \delta 1.03(\mathrm{t}, J=7.0 \mathrm{~Hz}, 6 \mathrm{H}), 2.56-$ 2.59 (m, 4 H), 2.68-2.70 (m, $2 \mathrm{H}), 3.32-3.33$ (m, $2 \mathrm{H}), 5.15$ (br. s, $1 \mathrm{H}), 6.41(\mathrm{~d}, J=7.5 \mathrm{~Hz}, 1 \mathrm{H}), 6.54(\mathrm{~d}, J=7.5 \mathrm{~Hz}, 1 \mathrm{H}), 7.40(\mathrm{t}, J$ $=7.5 \mathrm{~Hz}, 1 \mathrm{H}), 8.10-8.11(\mathrm{~m}, 1 \mathrm{H})$.

${ }^{13} \mathrm{C} \mathrm{NMR}\left(150 \mathrm{MHz}, \mathrm{CDCl}_{3}\right) \delta 11.6,39.4,46.7,51.6,107.4$, $112.6,137.2,148.0,158.9$.

FT-IR (film, $\mathrm{cm}^{-1}$ ): 3322, 2954, 1668, 1454, 1152, 770, 732.

GC/MS (70 eV) m/z (\%): $193\left(\mathrm{M}^{+}, 11\right), 135$ (6), 95 (6), 86 (100), 51 (5), 42 (8).

HRMS (ESI) $m / z$ calcd for $\left[\mathrm{C}_{11} \mathrm{H}_{19} \mathrm{~N}_{3}+\mathrm{H}\right]^{+}:$194.1652; found: 194.1653 .

\section{$\mathrm{N}$-Heptylaniline (3ca)}

Yellow oil, yield $97 \%$.

${ }^{1} \mathrm{H}$ NMR $\left(600 \mathrm{MHz}, \mathrm{CDCl}_{3}\right) \delta$ 0.89-0.91 (m, $\left.3 \mathrm{H}\right), 1.30-1.41$ (m, $6 \mathrm{H}), 1.60-1.65(\mathrm{~m}, 4 \mathrm{H}), 3.11(\mathrm{t}, J=7.0 \mathrm{~Hz}, 2 \mathrm{H}), 3.49$ (br. $\mathrm{s}$, $1 \mathrm{H}), 6.61(\mathrm{~d}, J=7.9 \mathrm{~Hz}, 2 \mathrm{H}), 6.70(\mathrm{t}, J=7.9 \mathrm{~Hz}, 1 \mathrm{H}), 7.18(\mathrm{t}, J$ $=7.9 \mathrm{~Hz}, 2 \mathrm{H})$.

${ }^{13} \mathrm{C} \mathrm{NMR}\left(150 \mathrm{MHz}, \mathrm{CDCl}_{3}\right) \delta 14.1,22.6,27.1,29.1,29.6,31.8$, 44.0, 112.7, 115.9, 129.2, 148.7 .

FT-IR (film, $\mathrm{cm}^{-1}$ ): 3400, 3010, 2850, 1678, 1450, 650, 780.

GC/MS (70 eV) m/z (\%): $191\left(\mathrm{M}^{+}, 15\right), 107(7), 106(100)$, 77 (10).

HRMS (ESI) $m / z$ calcd for $\left[\mathrm{C}_{13} \mathrm{H}_{21} \mathrm{~N}+\mathrm{H}\right]^{+}:$192.1747; found: 192.1749 .

\section{$\mathrm{N}$-Heptylpyridin-2-amine (3ck)}

Colorless oil, yield 98\%.

${ }^{1} \mathrm{H}$ NMR (600 MHz, $\left.\mathrm{CDCl}_{3}\right) \delta$ 0.89-0.91 (m, $\left.3 \mathrm{H}\right), 1.30-1.42$ (m, 6 H), 1.61-1.65 (m, 4H), 3.25-3.26 (m, $2 \mathrm{H}), 4.49$ (br. s, $1 \mathrm{H}), 6.41-6.42(\mathrm{~m}, 1 \mathrm{H}), 6.58-6.59(\mathrm{~m}, 1 \mathrm{H}), 7.42-7.45(\mathrm{~m}, 1 \mathrm{H})$, $8.11-8.12(\mathrm{~m}, 1 \mathrm{H})$.

${ }^{13} \mathrm{C} \mathrm{NMR}\left(150 \mathrm{MHz}, \mathrm{CDCl}_{3}\right) \delta 14.0,22.6,27.0,29.1,30.9,31.8$, 42.2, 105.6, 115.8, 137.3, 148.4, 159.0 .

FT-IR (film, $\mathrm{cm}^{-1}$ ): 3326, 2926, 1668, 1604, 1515, 1455, 1377, $1289,769$.

GC/MS (70 eV) m/z (\%): $192\left(\mathrm{M}^{+}, 13\right), 176(5), 121(26), 108$ (23), 107 (100), 94 (38), 78 (26), 41 (6).

HRMS (ESI) $m / z$ calcd for $\left[\mathrm{C}_{12} \mathrm{H}_{20} \mathrm{~N}_{2}+\mathrm{H}\right]^{+}:$193.1699; found: 193.1670 .

\section{$N$-Phenethylaniline (3da)}

Colorless oil, yield $85 \%$.

${ }^{1} \mathrm{H}$ NMR $\left(600 \mathrm{MHz}, \mathrm{CDCl}_{3}\right) \delta 2.93(\mathrm{t}, J=7.0 \mathrm{~Hz}, 2 \mathrm{H}), 3.41(\mathrm{t}$, $J=7.0 \mathrm{~Hz}, 2 \mathrm{H}$ ), 3.68 (br. s, $1 \mathrm{H}), 6.61-6.64$ (m, $2 \mathrm{H}), 6.70-6.73$ (m, 1 H), 7.16-7.21 (m, 2 H), 7.23-7.25 (m, 3H), 7.31-7.34 (m, $2 \mathrm{H})$.

${ }^{13} \mathrm{C} \mathrm{NMR}\left(150 \mathrm{MHz}, \mathrm{CDCl}_{3}\right) \delta 35.5,45.0,113.0,117.5,126.4$, 128.6, 128.8, 129.3, 139.3, 148.0.

FT-IR (film, $\mathrm{cm}^{-1}$ ): 3408, 3025, 2927, 2860, 1601, 1505, 1475, 1319, 1179, 1079, 1029, 869, 747.

GC/MS (70 eV) m/z (\%): $197\left(\mathrm{M}^{+}, 12\right), 107$ (8), 106 (100), 91 (8), 77 (18), 65 (5), 51 (5).

HRMS (ESI) $m / z$ calcd for $\left[\mathrm{C}_{14} \mathrm{H}_{15} \mathrm{~N}+\mathrm{H}\right]^{+}:$198.1277; found: 198.1278 . 


\section{$\mathrm{N}$-Cyclohexyl-4-methoxyaniline (3eb)}

White solid, m.p. $41-42^{\circ} \mathrm{C}$, yield $70 \%$.

${ }^{1} \mathrm{H}$ NMR (600 MHz, $\mathrm{CDCl}_{3}$ ) $\delta 1.01-1.37$ (m, 4 H), 1.60-2.06 $(\mathrm{m}, 6 \mathrm{H}), 3.47-3.50(\mathrm{~m}, 1 \mathrm{H}), 3.75(\mathrm{~s}, 3 \mathrm{H}), 6.57(\mathrm{~d}, J=8.8,2 \mathrm{H})$, $6.76(\mathrm{~d}, J=8.8,2 \mathrm{H})$.

${ }^{13} \mathrm{C} \mathrm{NMR}\left(150 \mathrm{MHz}, \mathrm{CDCl}_{3}\right) \delta 25.1,26.0,33.7,52.8,55.9$, $114.9_{1}, 114.9_{3}, 141.6,151.8$.

FT-IR (KBr, cm $\left.{ }^{-1}\right)$ : 3344, 3039, 3012, 2929, 2851, 1845, 1617, $1588,1515,1468,1445,1410,1365,1291,1244,1227,1184,1145$, $1117,1092,1033,977,886,819,753,650,624,557,511,466$.

GC/MS (70 eV) m/z (\%): $205\left(\mathrm{M}^{+}, 66\right), 163(12), 162$ (100), 149 (17), 134 (13), 108 (22), 77 (5), 55 (5), 41 (6).

HRMS (ESI) $m / z$ calcd for $\left[\mathrm{C}_{13} \mathrm{H}_{19} \mathrm{NO}+\mathrm{H}\right]^{+}:$206.1539; found: 206.1540 .

\section{$\mathrm{N}$-Cyclohexylpyridin-2-amine (3ek)}

White solid, m.p. $106-107^{\circ} \mathrm{C}$, yield $53 \%$.

${ }^{1} \mathrm{H}$ NMR $\left(600 \mathrm{MHz} \mathrm{CDCl}_{3}\right) \delta 1.32-1.40(\mathrm{~m}, 3 \mathrm{H}), 1.54-1.63$ (m, 3 H), 1.85-1.88 (m, 3 H), 1.99-2.00 (m, 2 H), 3.50-3.51 (m, $1 \mathrm{H}), 6.69-6.75(\mathrm{~m}, 1 \mathrm{H}), 6.78-6.81(\mathrm{~m}, 1 \mathrm{H}), 7.75-7.79(\mathrm{~m}, 2 \mathrm{H})$.

${ }^{13} \mathrm{C}$ NMR $\left(150 \mathrm{MHz}, \mathrm{CDCl}_{3}\right) \delta 24.3,25.1,32.1,51.7,108.0$, $111.0,136.3,143.7,153.0$.

FT-IR (KBr, cm ${ }^{-1}$ ): 3265, 3143, 3091, 3069, 3020, 2924, 2852, $1610,1573,1520,1486,1452,1416,1362,1345,1285,1251,1227$, 1152, 1116, 1095, 987, 974, 891, 861, 766, 731, 682, 580, 524.

GC/MS (70 eV) m/z (\%): $176\left(\mathrm{M}^{+}, 48\right), 147$ (11), $133(44), 120$ (10), 119 (100), 107 (8), 98 (9), 95 (12), 79 (11), 67 (12), 52 (5), 41 (6).

HRMS (ESI) $m / z$ calcd for $\left[\mathrm{C}_{11} \mathrm{H}_{16} \mathrm{~N}_{2}+\mathrm{H}\right]^{+}:$177.1386; found: 177.1387 .

\section{1-[2-(Cyclohexylamino)phenyl]ethan-1-one (3el)}

Colorless oil, yield $60 \%$.

${ }^{1} \mathrm{H}$ NMR $\left(600 \mathrm{MHz}, \mathrm{CDCl}_{3}\right) \delta 1.28-1.41(\mathrm{~m}, 5 \mathrm{H}), 1.62-1.64$ (m, $1 \mathrm{H}), 1.78-1.80(\mathrm{~m}, 2 \mathrm{H}), 2.01-2.02(\mathrm{~m}, 2 \mathrm{H}), 2.58(\mathrm{~s}, 3 \mathrm{H})$, $3.42-3.46(\mathrm{~m}, 1 \mathrm{H}), 6.53(\mathrm{t}, J=8.0 \mathrm{~Hz}, 1 \mathrm{H}), 6.74(\mathrm{~d}, J=8.0 \mathrm{~Hz}$, $1 \mathrm{H}), 7.32(\mathrm{t}, J=8.0 \mathrm{~Hz}, 1 \mathrm{H}), 7.74(\mathrm{~d}, J=8.0 \mathrm{~Hz}, 1 \mathrm{H})$.

${ }^{13} \mathrm{C}$ NMR $\left(150 \mathrm{MHz}, \mathrm{CDCl}_{3}\right) \delta 24.5,25.8,29.6,32.7,50.3$, $112.1,113.3,117.2,132.9,134.8,150.2,200.6$.

FT-IR (film, $\mathrm{cm}^{-1}$ ): 3290, 2927, 2851, 1607, 1574, 1519, 1459, 1421, 1333, 1258, 1230, 1161, 1098, 1036, 952, 888, 802, 744.

GC/MS (70 eV) m/z (\%): $217\left(\mathrm{M}^{+}, 90\right), 202(36), 200(24), 188$ (11), 174 (24), 162 (15), 160 (269), 146 (19), 136 883), 121 (14), 120 (100), 107 (11), 97 (14), 91 (42), 65 (18), 55 (24), 41 (22).

HRMS (ESI) $m / z$ calcd for $\left[\mathrm{C}_{14} \mathrm{H}_{19} \mathrm{NO}+\mathrm{H}\right]^{+}: 218.1539$; found: 218.1540 .

\section{$\mathbf{N}, \mathbf{N}$-Dibutylpyridin-2-amine (3fk)}

Colorless liquid, yield $80 \%$.

${ }^{1} \mathrm{H}$ NMR $\left(600 \mathrm{MHz}, \mathrm{CDCl}_{3}\right) \delta 0.95(\mathrm{t}, J=7.3 \mathrm{~Hz}, 6 \mathrm{H}), 1.33-$ $1.38(\mathrm{~m}, 4 \mathrm{H}), 1.56-1.60(\mathrm{~m}, 4 \mathrm{H}), 3.42(\mathrm{t}, J=7.6 \mathrm{~Hz}, 4 \mathrm{H})$, 6.41-6.46 (m, $2 \mathrm{H}), 7.36-7.39$ (m, $1 \mathrm{H}), 8.11-8.12(\mathrm{~m}, 1 \mathrm{H})$.

${ }^{13} \mathrm{C}$ NMR $\left(150 \mathrm{MHz}, \mathrm{CDCl}_{3}\right) \delta 14.2,20.3,29.9,48.8,105.6$, $110.5,136.9,148.1,157.7$.

FT-IR (film, $\mathrm{cm}^{-1}$ ): 3370, 2923, 2852, 1738, 1596, 1463, 1376.

GC/MS (70 eV) m/z (\%): 206 (30), 177 (9), 163 (51), 149 (18), 121 (100), 119 (10), 107 (81),78 (31), 41 (10).
HRMS (ESI) $m / z$ calcd for $\left[\mathrm{C}_{13} \mathrm{H}_{22} \mathrm{~N}_{2}+\mathrm{H}\right]^{+}:$207.1856; found: 207.1857 .

\section{4-Phenylmorpholine (3ga)}

Colorless solid, m.p. $51-52^{\circ} \mathrm{C}$, yield $90 \%$.

${ }^{1} \mathrm{H}$ NMR (600 MHz, $\mathrm{CDCl}_{3}$ ) $\delta$ 3.17-3.18 (m, $\left.4 \mathrm{H}\right)$ 3.87-3.89 (m, $4 \mathrm{H}), 6.89-6.94(\mathrm{~m}, 2 \mathrm{H}), 7.25-7.32(\mathrm{~m}, 3 \mathrm{H})$.

${ }^{13} \mathrm{C} \mathrm{NMR}\left(150 \mathrm{MHz}, \mathrm{CDCl}_{3}\right) \delta 49.2,67.8,115.8,120.1$, 129.4, 151.6.

FT-IR $\left(\mathrm{KBr}, \mathrm{cm}^{-1}\right)$ 3057, 3023, 3002, 2962, 2888, 2855, 2825, 2761, 2687, 1973, 1598, 1494, 1447, 1376, 1298, 1260, 1228, 1176, 1119, 1064, 1049, 1031, 990, 924, 858, 772, 698, 636, 519.

GC/MS (70 eV) m/z (\%): $163\left(\mathrm{M}^{+}, 53\right), 132(6), 105$ (100), 104 (43), 77 (27), 51 (8).

HRMS (ESI) $m / z$ calcd for $\left[\mathrm{C}_{10} \mathrm{H}_{13} \mathrm{NO}+\mathrm{H}\right]^{+}: 164.1070$; found: 164.1071 .

\section{4-(Pyridin-2-yl)morpholine (3gk)}

Colorless oil, yield 90\%.

${ }^{1} \mathrm{H}$ NMR $\left(600 \mathrm{MHz}, \mathrm{CDCl}_{3}\right) \delta 3.51-3.52(\mathrm{~m}, 4 \mathrm{H}), 3.83-$ $3.85(\mathrm{~m}, 4 \mathrm{H}), \quad 6.64-6.68(\mathrm{~m}, 2 \mathrm{H}), \quad 7.49-7.52(\mathrm{~m}, 1 \mathrm{H})$, $8.21-8.22(\mathrm{~m}, 1 \mathrm{H})$.

${ }^{13} \mathrm{C} \mathrm{NMR}\left(150 \mathrm{MHz}, \mathrm{CDCl}_{3}\right) \delta 45.6,66.7,106.9,113.7,137.4$, 147.9, 159.6.

FT-IR (film, $\mathrm{cm}^{-1}$ ): 3476, 2962, 2892, 2852, 1722, 1593, 1563, $1481,1436,1376,1333,1311,1242,1160,1120,1069,950,942$, $855,775,734,645,615,528,457$.

GC/MS (70 eV) m/z (\%): $164\left(\mathrm{M}^{+}, 36\right), 163(28), 119(23), 107$ (39), 106 (14), 79 (100), 78 (27), 52 (12), 51 (12).

HRMS (ESI) $m / z$ calcd for $\left[\mathrm{C}_{9} \mathrm{H}_{12} \mathrm{~N}_{2} \mathrm{O}+\mathrm{H}\right]^{+}: 165.1022$; found: 165.1021 .

\section{Typical Procedure for the Ullmann Cross-Coupling Reaction With Aromatic Amines: Synthesis of Diphenylamine 5aa}

CuI (10 mol\%, $0.05 \mathrm{mmol}, 10 \mathrm{mg})$, iodobenzene (1n, 1 equiv, 0.5 mmol, $102 \mathrm{mg}, 57 \mu \mathrm{L}$ ), aniline (4a, 1 equiv, $0.5 \mathrm{mmol}, 47 \mathrm{mg}$, $46 \mu \mathrm{L})$ and the base ( $t$-BuOK, 2 equiv, $1 \mathrm{mmol}, 112 \mathrm{mg}$ ) were suspended in $1 \mathrm{~g}$ DES (Gly/ChCl, 2:1 mol mol${ }^{-1}$ ), under air, in a vial with a Teflon screw tap under vigorous stirring at $100^{\circ} \mathrm{C}$. The reaction was monitored by GC. Then, the mixture was extracted with CPME $(3 \times 1 \mathrm{~mL})$ and the organic phase was dried over anhydrous $\mathrm{Na}_{2} \mathrm{SO}_{4}$ and filtered over a celite pad. Evaporation of the solvent under reduced pressure afforded the crude that was purified by flash-chromatography on silica gel (hexane/AcOEt $8: 2$ ) to provide the desired product 5 aa in $98 \%$ yield $(83 \mathrm{mg})$.

\section{Diphenylamine (5aa)}

White solid, $98 \%$.

${ }^{1} \mathrm{H}$ NMR $\left(600 \mathrm{MHz}, \mathrm{CDCl}_{3}\right) \delta 5.70$ (br. s, $\left.1 \mathrm{H}\right), 6.93-6.96(\mathrm{~m}$, $2 \mathrm{H}), 7.08-7.10$ (m, $4 \mathrm{H}), 7.27-7.29(\mathrm{~m}, 4 \mathrm{H})$.

${ }^{13} \mathrm{C} \mathrm{NMR}\left(150 \mathrm{MHz}, \mathrm{CDCl}_{3}\right) \delta 117.8,121.0,129.3,143.2$.

FT-IR (film, $\mathrm{cm}^{-1}$ ): 3380, 3034, 1583, 1489, 739, 685.

GC/MS (70 eV) m/z (\%): $169\left(\mathrm{M}^{+}, 100\right), 168(64), 167$ (36), 141 (5), 115 (6), 77 (8), 65 (6), 51 (9).

HRMS (ESI) $m / z$ calcd for $\left[\mathrm{C}_{12} \mathrm{H}_{11}+\mathrm{H}\right]^{+}:$170.0964; found: 170.0965 . 


\section{3-Methoxy- $N$-phenylaniline (5ac)}

Yellow oil, yield $70 \%$.

${ }^{1} \mathrm{H}$ NMR $\left(600 \mathrm{MHz}, \mathrm{CDCl}_{3}\right) \delta 3.80$ (s, $\left.3 \mathrm{H}\right), 5.74$ (br. s, $1 \mathrm{H}), 6.50-6.53(\mathrm{~m}, 1 \mathrm{H}), 6.66-6.68(\mathrm{~m}, 2 \mathrm{H}), 6.95-6.98(\mathrm{~m}, 1 \mathrm{H})$,

7.11-7.12 (m, $2 \mathrm{H}), 7.17-7.20$ (m, $1 \mathrm{H}), 7.27-7.31$ (m, $2 \mathrm{H})$.

${ }^{13} \mathrm{C} \mathrm{NMR}\left(150 \mathrm{MHz}, \mathrm{CDCl}_{3}\right) \delta 55.2,103.3,106.2,110.2,118.4$,

$121.3,129.4,130.1,142.8,144.6,160.7$.

FT-IR (film, $\mathrm{cm}^{-1}$ ): 3379, 3029, 1638, 1517, 1464, 1296, $1177,870$.

GC/MS (70 eV) m/z (\%): $199\left(\mathrm{M}^{+}, 100\right), 183(9), 168$ (16), 167 (13), 156 (7), 155 (8), 128 (12), 51 (7).

HRMS (ESI) $m / z$ calcd for $\left[\mathrm{C}_{13} \mathrm{H}_{13} \mathrm{NO}+\mathrm{H}\right]^{+}: 200.1070$; found: 200.1071 .

\section{4-Methoxy-N-phenylaniline (5ao)}

Yellow oil, yield $70 \%$.

${ }^{1} \mathrm{H}$ NMR (600 MHz, $\left.\mathrm{CDCl}_{3}\right) \delta 3.81$ (s, $3 \mathrm{H}$ ), 4.69 (br. s, $1 \mathrm{H}), 6.86-6.88(\mathrm{~m}, 3 \mathrm{H}), 6.91-6.93(\mathrm{~m}, 2 \mathrm{H}), 7.08-7.10(\mathrm{~m}, 2 \mathrm{H})$, 7.21-7.24 (m, $2 \mathrm{H})$.

${ }^{13} \mathrm{C} \mathrm{NMR}\left(150 \mathrm{MHz}, \mathrm{CDCl}_{3}\right) \delta 55.6,114.7,115.7,119.6,122.2$, $129.3,130.9,135.8,145.1$.

FT-IR (film, $\mathrm{cm}^{-1}$ ): 3408, 2936, 2834, 1590, 1512, 1495, 1464, 1419, 1295, 1231, 1176, 1114, 1025, 908.

GC/MS $(70 \mathrm{eV}) \mathrm{m} / z(\%): 199\left(\mathrm{M}^{+}, 64\right), 185$ (13), 184 (100), 154 (10), 129 (10), 128 (13), 78 (11), 51 (7).

HRMS (ESI) $m / z$ calcd for $\left[\mathrm{C}_{13} \mathrm{H}_{13} \mathrm{NO}+\mathrm{H}\right]^{+}: 200.1070$; found: 200.1073 .

\section{3-Hydroxydiphenylamine (5ap)}

Brown solid, m. p. $70^{\circ} \mathrm{C}$, yield $90 \%$.

${ }^{1} \mathrm{H}$ NMR $\left(600 \mathrm{MHz}, \mathrm{CDCl}_{3}\right) \delta 5.33$ (br. s, $1 \mathrm{H}$ ), 5.71 (br. s, $1 \mathrm{H}), 6.39-6.40(\mathrm{~m}, 1 \mathrm{H}), 6.58-6.62(\mathrm{~m}, 2 \mathrm{H}), 6.94-6.97$ (m, $1 \mathrm{H})$, 7.09-7.12 (m, $3 \mathrm{H}), 7.26-7.29$ (m, $2 \mathrm{H})$.

${ }^{13} \mathrm{C}$ NMR $\left(150 \mathrm{MHz}, \mathrm{CDCl}_{3}\right) \delta 104.0,107.7,110.0,118.7$, $121.5,129.3,130.3,142.6,145.0,156.6$.

FT-IR (KBr, cm $\left.{ }^{-1}\right)$ : 3378, 1599, 1507, 1457, 1412, 1318, 1238, 1102, 822, 744, 693, 511.

GC/MS (70 eV) m/z (\%): $185\left(\mathrm{M}^{+}, 100\right), 166(10), 156(9), 154$ (8), 129 (6), 77 (7).

HRMS (ESI) $m / z$ calcd for $\left[\mathrm{C}_{12} \mathrm{H}_{11} \mathrm{NO}+\mathrm{H}\right]^{+}:$186.0913; found: 186.0914 .

\section{2-Methyl-5-(phenylamino)benzonitrile (5aq)}

Yellow oil, yield $70 \%$.

${ }^{1} \mathrm{H}$ NMR $\left(600 \mathrm{MHz}, \mathrm{CDCl}_{3}\right) \delta 2.48(\mathrm{~s}, 3 \mathrm{H}), 6.98-7.07$ (m, $3 \mathrm{H}), 7.14-7.19$ (m, $2 \mathrm{H}), 7.29-7.33$ ( $\mathrm{m}, 3 \mathrm{H})$.

${ }^{13} \mathrm{C} \mathrm{NMR}\left(150 \mathrm{MHz}, \mathrm{CDCl}_{3}\right) \delta 19.6,114.6,118.7,120.0,121.3$, $121.9,122.3,129.6,131.2,133.7,141.9$.

FT-IR (film, cm ${ }^{-1}$ ): 3368, 2918, 1605, 1463, 1261, 1047.

GC/MS (70 eV) m/z (\%): $208\left(\mathrm{M}^{+} 100\right), 207$ (56), 192 (17), 152 (5), 103 (5), 77 (10), 51 (6).

HRMS (ESI) $m / z$ calcd for $\left[\mathrm{C}_{14} \mathrm{H}_{12} \mathrm{~N}_{2}+\mathrm{H}\right]^{+}:$209.1073; found: 209.1074 .

\section{4-Ethyl- $N$-phenylaniline (5bn)}

Yellow solid, m.p.: 86-87, yield 90\%.

${ }^{1} \mathrm{H} \mathrm{NMR}\left(600 \mathrm{MHz}, \mathrm{CDCl}_{3}\right) \delta 1.22(\mathrm{t}, J=7.1 \mathrm{~Hz}, 3 \mathrm{H})$, $2.62(\mathrm{q}, J=7.1 \mathrm{~Hz}, 2 \mathrm{H}), 5.63$ (br. s, $1 \mathrm{H}), 6.89(\mathrm{t}, J=$
$7.5 \mathrm{~Hz}, 1 \mathrm{H}), 7.03-7.05(\mathrm{~m}, 4 \mathrm{H}), 7.12$ (d, $J=7.5 \mathrm{~Hz}, 2 \mathrm{H}), 7.25$ $(\mathrm{d}, J=7.5 \mathrm{~Hz}, 2 \mathrm{H})$.

${ }^{13} \mathrm{C} \mathrm{NMR}\left(150 \mathrm{MHz}, \mathrm{CDCl}_{3}\right) \delta 15.3,29.7,117.0,118.8,120.3$, 128.7, 129.3, 134.5, 141.4, 144.3.

FT-IR (film, $\mathrm{cm}^{-1}$ ): 3393, 3052, 3032, 2962, 2924, 2869, 1596, $1514,1442,1400,1313,1235,1173,1115,1075,1046,1028,994$, $876,847,818,743,692,615,549,506$.

GC/MS (70 eV) m/z (\%): $197\left(\mathrm{M}^{+}, 43\right), 183(15), 182(100)$, 180 (7), 167 (12), 90 (5), 77 (8).

HRMS (ESI) $m / z$ calcd for $\left[\mathrm{C}_{14} \mathrm{H}_{15} \mathrm{~N}+\mathrm{H}\right]^{+}:$198.1277; found: 198.1278 .

\section{4-Ethyl-N-(4-methoxyphenyl)aniline (5bo)}

Colorless oil, yield $97 \%$.

${ }^{1} \mathrm{H}$ NMR $\left(600 \mathrm{MHz}_{\mathrm{CDCl}}\right) \delta 1.22(\mathrm{t}, J=6.7 \mathrm{~Hz}, 3 \mathrm{H}), 2.58$ (q, $J=6.7 \mathrm{~Hz}, 2 \mathrm{H}), 3.80$ (s, $3 \mathrm{H}), 5.40$ (br. s, $1 \mathrm{H}), 6.85-6.89$ (m, $4 \mathrm{H}), 7.03-7.07$ (m, $4 \mathrm{H})$.

${ }^{13} \mathrm{C} \mathrm{NMR}\left(150 \mathrm{MHz}, \mathrm{CDCl}_{3}\right) \delta 15.8,28.0,55.6,114.7,116.5$, 121.2, 128.6, 135.9, 136.6, 142.6, 154.8.

FT-IR (film, $\mathrm{cm}^{-1}$ ): 3390, 2924, 2853, 1613, 1463, 1295, 1243, $1180,1038,821$.

GC/MS (70 eV) m/z (\%): $227\left(\mathrm{M}^{+}, 62\right), 213(16), 212(100)$, 197 (10), 168 (11), 77 (5).

HRMS (ESI) $\mathrm{m} / \mathrm{z}$ calcd for $\left[\mathrm{C}_{15} \mathrm{H}_{17} \mathrm{NO}+\mathrm{H}\right]^{+}: 228.1383$; found: 228.1384 .

\section{3,5-Dimethyl- $N$-phenylaniline $(5 \mathrm{cn})$}

Yellow oil, yield $60 \%$.

${ }^{1} \mathrm{H} \mathrm{NMR}\left(600 \mathrm{MHz}, \mathrm{CDCl}_{3}\right) \delta 2.29$ (s, 6 H), 5.62 (br. s, $\left.1 \mathrm{H}\right)$, $6.61(\mathrm{~s}, 1 \mathrm{H}), 6.73(\mathrm{~s}, 2 \mathrm{H}), 6.93(\mathrm{t}, J=7.5 \mathrm{~Hz}, 1 \mathrm{H}), 7.07$ (d, $J=$ $7.5 \mathrm{~Hz}, 2 \mathrm{H}), 7.28(\mathrm{t}, J=7.5 \mathrm{~Hz}, 2 \mathrm{H})$.

${ }^{13} \mathrm{C} \mathrm{NMR}\left(150 \mathrm{MHz}, \mathrm{CDCl}_{3}\right) \delta 21.4,115.6,117.9,120.8,122.9$, 129.3, 139.0, 143.1, 143.3.

FT-IR (film, $\mathrm{cm}^{-1}$ ): 3383, 3025, 2914, 1583, 1516, 1496, 1327, 1265, 1169, 1081, 1034, 992, 878, 843.

GC/MS (70 eV) m/z (\%): $197\left(\mathrm{M}^{+}, 100\right), 181(21), 180$ (16), 167 (11), 164 (8), 121 (18), 120 (10), 106 (11), 91 (8), 77 (14), 65 (6).

HRMS (ESI) $m / z$ calcd for $\left[\mathrm{C}_{14} \mathrm{H}_{15} \mathrm{~N}+\mathrm{H}\right]^{+}:$198.1277; found: 198.1278 .

\section{Recycling of Cu Catalyst, DES and Base in the Coupling Reaction Between Bromobenzene (1a) and $\mathrm{N}, \mathrm{N}$-dimethylethylenediamine (2a) to Prepare 3aa. Typical Procedure}

CuI (10 mol\%, $0.05 \mathrm{mmol}, 10 \mathrm{mg}$ ), bromobenzene (1a, 1 equiv, $0.5 \mathrm{mmol}, 79 \mathrm{mg}, 53 \mu \mathrm{L}), \mathrm{N}, \mathrm{N}$-dimethylethylenediamine (2a, 1 equiv, $0.5 \mathrm{mmol}, 44 \mathrm{mg}, 55 \mu \mathrm{L})$ and the base $\left(\mathrm{K}_{2} \mathrm{CO}_{3}, 2\right.$ equiv, 1 $\mathrm{mmol}, 138 \mathrm{mg}$ ) were sequentially added in $1.0 \mathrm{~g} \mathrm{DES}(\mathrm{Gly} / \mathrm{ChCl}$, 2:1 $\mathrm{mol} \mathrm{mol}^{-1}$ ), under air, in a vial with a Teflon screw tap. The reaction mixture was vigorously stirred at $60^{\circ} \mathrm{C}$ for $12 \mathrm{~h}$ (monitoring the complete consumption of the starting material by GC), and then cooled to room temperature. The product was extracted with CPME $(1 \mathrm{~mL})$ leaving the catalyst and the base in the eutectic mixture, which was re-used for further reaction runs. The organic layer was filtered through a celite pad, the volatile was evaporated under vacuum, and the crude so obtained was analyzed by ${ }^{1} \mathrm{H}$ NMR to determine the yield of $\mathbf{3 a a}\left(\mathrm{CH}_{2} \mathrm{Br}_{2}\right.$ was 
used as the internal standard). New, fresh reagents were then added to the recovered eutectic mixture, and the whole procedure was repeated for five times without any significant loss of the catalyst activity (96\% yield after the sixth cycle) (see Figure 2 ). By analyzing the $\mathrm{Cu}$ amount in the CPME extract phase by ICPOES, we conclude that up to $2 \%$ of the total amount of copper in the catalyst was lost during each extraction process.

\section{DATA AVAILABILITY STATEMENT}

All datasets generated for this study are included in the article/Supplementary Material.

\section{AUTHOR CONTRIBUTIONS}

AQ undertook all the synthetic experimental works. AQ and FP analyzed and collected experimental data. VC directed the project. PV, FP, and VC supervised the synthetic chemistry works. PV and FP carried out the characterization chemistry works. VC analyzed and drafted the manuscript, while AQ, PV, FP, and VC contributed to the discussion, to the writing, and the review.

\section{FUNDING}

This work was carried out within the framework of the Programma Operativo Nazionale Ricerca e Innovazione

\section{REFERENCES}

Alonso, D. A., Baeza, A., Chinchilla, R., Guillena, G., Pastor, I. M., and Ramón, D. J. (2016). Deep eutectic solvents: the organic reaction medium of the century. Eur. J. Org. Chem. 2016, 612-632. doi: 10.1002/ejoc.201501197

Azzena, U., Carraro, M., Pisano, L., Monticelli, S., Bartolotta, R., and Pace, V. (2019). Cyclopentyl methyl ether: an elective ecofriendly ethereal solvent in classical and modern organic chemistry. ChemSusChem 12, 40-70. doi: $10.1002 /$ cssc. 201801768

Beletskaya, I. P., and Ananikov, V. P. (2011). Transition-metal-catalyzed C-S, C$\mathrm{Se}$, and C-Te bond formation via cross-coupling and atom-economic addition reactions. Chem. Rev. 111, 1596-1636. doi: 10.1021/cr100347k

Calò, V., Nacci, A., Monopoli, A., and Cotugno, P. (2009). Palladiumnanoparticle-catalysed Ullmann reactions in ionic liquids with aldehydes as the reductants: scope and mechanism. Chem. Eur. J. 15, 1272-1279. doi: $10.1002 /$ chem.200801621

Casitas, A., and Ribas, X. (2013). The role of organometallic copper(III) complexes in homogeneous catalysis. Chem. Sci. 4, 2301-2318. doi: 10.1039/c3sc21818j

Chakraborti, G., Paladhi, S., Mandal, T., and Dash, J. (2018). “On water" promoted Ullmann-type C-N bond-forming reactions: application to carbazole alkaloids by selective $\mathrm{N}$-arylation of aminophenols. J. Org. Chem. 83, 7347-7359. doi: 10.1021 /acs.joc.7b03020

Chen, Y.-J., and Chen, H.-H. (2006). 1,1,1-Tris(hydroxymethyl)ethane as a new, efficient, and versatile tripod ligand for copper-catalyzed cross-coupling reactions of aryl iodides with amides, thiols, and phenols. Org. Lett. 8, 5609-5612. doi: 10.1021/ol062339h

Cheng, J., Tang, L., and $\mathrm{Xu}$, J. (2010). An economical, green pathway to biaryls: palladium nanoparticles catalyzed Ullmann reaction in ionic liquid/supercritical carbon dioxide system. Adv. Synth. Catal. 352, 3275-3286. doi: $10.1002 /$ adsc. 201000475

Cicco, L, Rodríguez-Álvarez, M. J., Perna, F. M., García-Álvarez, J., and Capriati, V. (2017). One-pot sustainable synthesis of tertiary alcohols by combining ruthenium-catalysed isomerisation of allylic alcohols and chemoselective
(PON RI 2014/2020), Axis I Investments in Human Capital, Action I.1.-Innovative Ph.D.s with industrial characterization, funding FSE-FESR (D.D. n. 1377 on 5/6/2017), and was financially supported by the national PRIN project "Unlocking Sustainable Technologies Through Nature-Inspired Solvents" (Code: 2017A5HXFC_002), by the Interuniversity Consortium C.I.N.M.P.I.S., and by the University of Bari Aldo Moro (codes: CapriatiV.FABR2018SFARMA, PernaF.FABR2018SFARMA, PernaF.18 FondiAteneo15-16, VitaleP.18 FondiAteno15-16, VitaleP.FABR2018SFARMA).

\section{ACKNOWLEDGMENTS}

The authors wish to thank Dr. Galatea Trotta for her contribution to the experimental work, and are also indebted to Professor Francesco Sannicolò (Laboratori Alchemia srl, Milan) for valuable discussions and comments, to Zeon Europe $\mathrm{GmbH}$ for the generous gift of a sample of CPME, and to Dr. Natale I. Volpicella and Dr. Damiano A. P. Manigrassi of AER Consulting for performing ICP-OES analysis.

\section{SUPPLEMENTARY MATERIAL}

The Supplementary Material for this article can be found online at: https://www.frontiersin.org/articles/10.3389/fchem. 2019.00723/full\#supplementary-material

addition of polar organometallic reagents in deep eutectic solvents. Green Chem. 19, 3069-3077. doi: 10.1039/C7GC00458C

Cicco, L., Ríos-Lombardía, N., Rodríguez-Álvarez, M. J., Morís, F., Perna, F. M., Capriati, V., et al. (2018). Programming cascade reactions interfacing biocatalysis with transition-metal catalysis in deep eutectic solvents as biorenewable reaction media. Green Chem. 20, 3468-3475. doi: 10.1039/C8GC00861B

Cicco, L., Sblendorio, S., Mansueto, R., Perna, F. M., Salomone, A., Florio, S., et al. (2016). Water opens the door to organolithiums and Grignard reagents: exploring and comparing the reactivity of highly polar organometallic compounds in unconventional reaction media towards the synthesis of tetrahydrofurans. Chem. Sci. 7, 1192-1199. doi: 10.1039/C5SC03436A

Cristau, H.-J., Cellier, P. P., Spindler, J.-F., and Taillefer, M. (2004). Highly efficient and mild copper-catalyzed $\mathrm{N}$ - and $\mathrm{C}$-arylations with aryl bromides and iodides. Chem. Eur. J. 10, 5607-5622. doi: 10.1002/chem.200400582

Cristau, H.-J., Ouali, A., Spindler, J.-F., and Taillefer, M. (2005). Mild and efficient copper-catalyzed cyanation of aryl iodides and bromides. Chem. Eur. J. 11, 2483-2492. doi: 10.1002/chem.200400979

De Meijere, A., and Diederich, F. (2004). Metal-Catalyzed Cross-Coupling Reactions. Weinheim: Wiley-VCH. doi: 10.1002/9783527619535

Dilauro, G., Dell'Aera, M., Vitale, P., Capriati, V., and Perna, F. M. (2017). Unprecedented nucleophilic additions of highly polar organometallic compounds to imines and nitriles using water as a non-innocent reaction medium. Angew. Chem. Int. Ed. Engl. 56, 10200-10203. doi: 10.1002/anie.201705412

Dilauro, G., Garcia, S. M., Tagarelli, D., Vitale, P., Perna, F. M., and Capriati, V. (2018). Ligand-free bioinspired Suzuki-Miyaura coupling reactions using aryltrifluoroborates as effective partners in deep eutectic solvents. ChemSusChem. 11, 3495-3501. doi: 10.1002/cssc.2018 01382

Dilauro, G., Quivelli, A. F., Vitale, P., Capriati, V., and Perna, F. M. (2019). Water and sodium chloride: essential ingredients for robust and fast Pd-catalysed cross-coupling reactions between organolithium reagents 
and (hetero)aryl halides. Angew. Chem. Int. Ed. Engl. 58, 1799-1802. doi: 10.1002/anie.201812537

Durán Pachón, L., Elsevier, C. J., and Rothenberg, G. (2006). Electroreductive palladium-catalysed Ullmann reactions in ionic liquids: scope and mechanism. Adv. Synth. Catal. 348, 1705-1710. doi: 10.1002/adsc.200606132

Evano, G., Blanchard, N., and Toumi, M. (2008). Copper-mediated coupling reactions and their applications in natural products and designed biomolecules synthesis. Chem. Rev. 108, 3054-3131. doi: 10.1021/cr8002505

Ferlin, F., Trombettoni, V., Luciani, L., Fusi, S., Piermatti, O., Santoro, S., et al. (2018). A waste-minimized protocol for copper-catalyzed Ullmann-type reaction in a biomass derived furfuryl alcohol/water azeotrope. Green Chem. 20, 1634-1639. doi: 10.1039/C8GC00287H

Gao, J., Bhunia, S., Wang, K., Gan, L., Xia, S., and Ma, D. (2017). Discovery of $\mathrm{N}$-(naphthalen-1-yl)- $\mathrm{N}^{\prime}$-alkyl oxalamide ligands enables $\mathrm{Cu}$ catalyzed aryl amination with high turnovers. Org. Lett. 19, 2809-2812. doi: 10.1021/acs.orglett.7b00901

García-Álvarez, J. (2015). Deep eutectic mixtures: promising sustainable solvents for metal-catalysed and metal-mediated organic reactions. Eur. J. Inorg. Chem. 2015, 5147-5157. doi: 10.1002/ejic.201500892

García-Álvarez, J., Hevia, E., and Capriati, V. (2015). Reactivity of polar organometallic compounds in unconventional reaction media: challenges and opportunities. Eur. J. Org. Chem. 2015, 6779-6799. doi: 10.1002/ejoc.201500757

García-Álvarez, J., Hevia, E., and Capriati, V. (2018). The future of polar organometallic chemistry written in bio-based solvents and water. Chem. Eur. J. 24, 14854-14863. doi: 10.1002/chem.201802873

Ge, X., Zhang, S., Chen, X., Liu, X., and Qian, C. (2019). A designed bi-functional sugar-based surfactant: micellar catalysis for $\mathrm{C}-\mathrm{X}$ coupling reaction in water. Green Chem. 21, 2771-2776. doi: 10.1039/C9GC00964G

Ghinato, S., Dilauro, G., Perna, F. M., Capriati, V., Blangetti, M., and Prandi, C. (2019). Directed ortho-metalation-nucleophilic acyl substitution strategies in deep eutectic solvents: the organolithium base dictates the chemoselectivity. Chem. Commun. 55, 7741-7744. doi: 10.1039/C9CC03927A

Giri, R., and Hartwig, J. F. (2010). Cu(I)-Amido complexes in the Ullmann reaction: reactions of $\mathrm{Cu}(\mathrm{I})$-amido complexes with iodoarenes with and without autocatalysis by CuI. J. Am. Chem. Soc. 132, 15860-15863. doi: $10.1021 /$ ja105695s

Goldberg, I. (1906). Ueber Phenylirungen bei Gegenwart von Kupfer als Katalysator. Ber. Dtsch. Chem. Ges. 39, 1691-1692. doi: 10.1002/cber.19060390298

Hartwig, J. F. (1998). Carbon-heteroatom bond-forming reductive eliminations of amines, ethers, and sulfides. Acc. Chem. Res. 31, 852-860. doi: 10.1021/ar970282g

Hartwig, J. F. (2000). Modern Amination Methods. Weinheim: Wiley-VCH.

Hayyan, M., Hashim, M. A., Hayyan, A., Al-Saadi, M. A., AlNashef, I. M., Mirghani, M. E., et al. (2013). Are deep eutectic solvents benign or toxic? Chemosphere 90, 2193-2195. doi: 10.1016/j.chemosphere.2012.11.004

Hayyan, M., Looi, C. Y., Hayyan, A., Wong, W. F., and Hashim M. A. (2015). In vitro and in vivo toxicity profiling of ammonium-based deep eutectic solvents. PLoS ONE 10:e117934. doi: 10.1371/journal.pone.0117934

Hayyan, M., Mbous, Y. P., Looi, C. Y., Wong, W. F., Hayyan, A., Salleh, Z., et al. (2016). Natural deep eutectic solvents: cytotoxic profile. SpringerPlus 29:913. doi: 10.1186/s40064-016-2575-9

Huang, L., Hu, R., Zhu, X., and Wan, Y. (2013). A recyclable Cu-catalyzed C-N coupling reaction in water and its application to synthesis of imidazo[1,2a] quinoxaline. Tetrahedron 69, 8974-8977. doi: 10.1016/j.tet.2013.07.036

Jiang, Y., Xu, L., Zhou, C., and Ma, D. (2013). "Cu-catalyzed Ullmann-type Cheteroatom bond formation: the key role of dinucleating ancillary ligands," in $\mathrm{C}-\mathrm{H}$ and $\mathrm{C}-\mathrm{X}$ Bond Functionalization: Transition Metal Mediation, ed X. Ribas (Cambridge: RSC Publishing), 1-45. doi: 10.1039/9781849737166-0 0001

Jiao, J., Zhang, X. R., Chang, N. H., Wang, J., Wei, J. F., Shi, X. Y., et al. (2011). A facile and practical copper powder-catalyzed, organic solvent- and ligand-free Ullmann amination of aryl halides. J. Org. Chem. 76, 1180-1183. doi: $10.1021 /$ jo102169t

Jones, C. P., Anderson, K. W., and Buchwald, S. L. (2007). Sequential Cucatalyzed amidation-base-mediated camps cyclization: a two-step synthesis of 2-aryl-4-quinolones from o-halophenones. J. Org. Chem. 72, 7968-7973. doi: $10.1021 /$ jo701384n
Li, X., Yang, D., Jiang, Y., and Fu, H. (2010). Efficient copper-catalyzed N-arylations of nitrogen-containing heterocycles and aliphatic amines in water. Green Chem. 12, 1097-1105. doi: 10.1039/c002172e

Lipshutz, B. H., Gallou, F., and Handa, S. (2016). Evolution of solvents in organic chemistry. ACS Sustain. Chem. Eng. 4, 5838-5849. doi: 10.1021/acssuschemeng.6b01810

Lipshutz, B. H., and Ghorai, S. (2014). Transitioning organic synthesis from organic solvents to water. What's your E factor? Green Chem. 16, 3660-3679. doi: 10.1039/C4GC00503A

Liu, P., Hao, J.-W., Mo, L.-P., and Zhang, Z.-H. (2015). Recent advances in the application of deep eutectic solvents as sustainable media as well as catalysts in organic reactions. RSC Adv. 5, 48685-48704. doi: 10.1039/C5RA05746A

Liu, S., and Zhou, J. (2013). Aqueous copper-catalyzed N-arylation of indoles: the surfactant strategy. New J. Chem. 37, 2537-2540. doi: 10.1039/c3nj00226h

Ma, D., and Cai, Q. (2008). Copper/amino acid catalyzed cross-couplings of aryl and vinyl halides with nucleophiles. Acc. Chem. Res. 41, 1450-1460. doi: $10.1021 /$ ar8000298

Mallardo, V., Rizzi, R., Sassone, F. C., Mansueto, R., Perna, F. M., Salomone, A., et al. (2014). Regioselective desymmetrization of diaryltetrahydrofurans via directed ortho-lithiation: an unexpected help from green chemistry. Chem. Commun. 50, 8655-8658. doi: 10.1039/C4CC03149K

Mancuso, R., Maner, A., Cicco, L., Perna, F. M., Capriati, V., and Gabriele, B. (2016). Synthesis of thiophenes in a deep eutectic solvent: heterocyclodehydration and iodocyclization of 1-mercapto-3-yn-2-ols in a choline chloride/glycerol medium. Tetrahedron 72, 4239-4244. doi: 10.1016/j.tet.2016.05.062

Martins, M. A. R., Pinho, S. P., and Coutinho, J. A. P. (2018). Insights into the nature of eutectic and deep eutectic mixtures. J. Solut. Chem. 48, 962-982. doi: 10.1007/s10953-018-0793-1

Messa, F., Perrone, S., Capua, M., Tolomeo, F., Troisi, L., Capriati, V., et al. (2018). Towards a sustainable synthesis of amides: chemoselective palladium-catalysed aminocarbonylation of aryl iodides in deep eutectic solvents. Chem. Commun. 54, 8100-8103. doi: 10.1039/C8CC03858A

Monnier, F., and Taillefer, M. (2009). Catalytic C-C, C-N, and C-O Ullmann-type coupling reactions. Angew. Chem. Int. Ed. 48, 6954-6971. doi: 10.1002/anie.200804497

Pant, P. L., Sonune, R. K., and Shankarling, G. S. (2018). Choline hydroxide promoted synthesis of $\mathrm{N}$-aryl anthraquinone derivatives: metal free approach to Ullmann coupling reactions. ChemistrySelect 3, 5249-5253. doi: 10.1002/slct.201800546

Pinto-Basto, D., Silva, J. P., Queiroz, M.-J. R. P., Moreno, A. J., and Coutinho, O. P. (2009). Antioxidant activity of synthetic diarylamines: A mitochondrial and cellular approach. Mitochondrion 9, 17-26. doi: 10.1016/j.mito.2008.10.001

Ribas, X., and Güell, I. (2014). Cu(I)/Cu(III) catalytic cycle involved in Ullmann-type cross-coupling reactions. Pure Appl. Chem. 86, 345-336. doi: 10.1515/pac-2013-1104

Roschangar, F., Sheldon, R. A., and Senenayake, C. (2015). Overcoming barriers to green chemistry in the pharmaceutical industry - the Green Aspiration Level $^{\text {TM }}$ concept. Green Chem. 17, 752-768. doi: 10.1039/C4GC01563K

Ruß, C., and König, B. (2012). Low melting mixtures in organic synthesis - an alternative to ionic liquids? Green Chem. 14, 2969-2982. doi: $10.1039 / \mathrm{c} 2 \mathrm{gc} 36005 \mathrm{e}$

Sambiagio, C., Marsden, S. P., Blacker, A. J., and McGowan, P. C. (2014). Copper catalysed Ullmann type chemistry: from mechanistic aspects to modern development. Chem. Soc. Rev. 43, 3525-3550. doi: 10.1039/C3CS60289C

Sánchez-Condado, A., Carriedo, G. A., Presa Soto, A., Rodríguez-Álvarez, M. J., García-Álvarez, J., and Hevia, E. (2019). Organolithium-initiated polymerization of olefins in deep eutectic solvents under aerobic conditions. ChemSusChem. 12, 3134-3143. doi: 10.1002/cssc.2019 00533

Sassone, F.C., Perna, F. M., Salomone, A., Florio, S., and Capriati, V. (2015). Unexpected lateral-lithiation-induced alkylative ring opening of tetrahydrofurans in deep eutectic solvents: synthesis of functionalised primary alcohols. Chem. Commun. 51, 9459-9462. doi: 10.1039/C5CC02884A

Seechurn, C. C. C. J., Kitching, M. O., Colacot, T. J., and Snieckus, V. (2012). Palladium-catalyzed cross-coupling: a historical contextual perspective to the 2010 nobel prize. Angew. Chem. Int. Ed. 51, 5062-5085. doi: 10.1002/anie. 201107017 
Shaabani, A., and Afshari, R. (2018). Magnetic Ugi-functionalized graphene oxide complexed with copper nanoparticles: efficient catalyst toward Ullman coupling reaction in deep eutectic solvents. J. Colloid Interf. Sci. 510, 384-394. doi: 10.1016/j.jcis.2017.09.089

Shafir, A., and Buchwald, S. L. (2006). Highly Selective room-temperature copper-catalyzed C-N coupling reactions. J. Am. Chem. Soc. 128, 8742-8743. doi: $10.1021 /$ ja063063b

Sheldon, R. A. (2007). The E factor: fifteen years on. Green Chem. 9, 1273-1283. doi: $10.1039 / \mathrm{b} 713736 \mathrm{~m}$

Sheldon, R. A. (2016). Biocatalysis and biomass conversion in alternative reaction media. Chem. Eur. J. 22, 12984-12999. doi: 10.1002/chem.201601940

Sherwood, J., Clark, J. H., Fairlamb, I. J. S., and Slattery, J. M. (2019). Solvent effects in palladium catalysed cross-coupling reactions. Green Chem. 21, 2164-2213. doi: 10.1039/C9GC00617F

Sperotto, E., van Klink, G. P. M., van Koten, G., and de Vries, J. G. (2010). The mechanism of the modified Ullmann reaction. Dalton Trans. 39, 10338-10351. doi: $10.1039 / \mathrm{c} 0 \mathrm{dt} 00674 \mathrm{~b}$

Strieter, E. R., Blackmond, D. G., and Buchwald, S. L. (2005). The role of chelating diamine ligands in the Goldberg Reaction: a kinetic study on the copper-catalyzed amidation of aryl iodides. J. Am. Chem. Soc. 127, 4120-4121. doi: $10.1021 /$ ja050120c

Surry, D. S., and Buchwald, S. L. (2008). Biaryl phosphane ligands in palladium-catalyzed amination. Angew. Chem. Int. Ed. 47, 6338-6361. doi: 10.1002/anie.200800497

Surry, D. S., and Buchwald, S. L. (2010). Diamine ligands in copper-catalyzed reactions. Chem. Sci. 1, 13-31. doi: 10.1039/c0sc00107d

Tye, J. W., Weng, Z., Johns, A. M., Incarvito, C. D., and Hartwig, J. F. (2008). Copper complexes of anionic nitrogen ligands in the amidation and imidation of aryl halides. J. Am. Chem. Soc. 130, 9971-9983. doi: 10.1021/ja076668w

Ullmann, F. (1901). Ueber Synthesen in der Biphenylreihe. Ber. Dtsch. Chem. Ges. 34, 2174-2185. doi: 10.1002/cber.190103402141

Ullmann, F. (1903). Ueber eine neue Bildungsweise von Diphenylaminderivaten. Ber. Dtsch. Chem. Ges. 36, 2382-2384. doi: 10.1002/cber.190303602174

Ullmann, F. (1904). Ueber eine neue Darstellungsweise von Phenyläthersalicylsäure. Ber. Dtsch. Chem. Ges. 37, 853-854. doi: 10.1002/cber.190403701141
Vidal, C., García-Álvarez, J., Hernán-Gómez, A., Kennedy, A. R., and Hevia, E. (2014). Introducing deep eutectic solvents to polar organometallic chemistry: chemoselective addition of organolithium and Grignard reagents to ketones in air. Angew. Chem. Int. Ed. Engl. 53, 5969-5973. doi: 10.1002/anie.201400889

Vidal, C., García-Álvarez, J., Hernán-Gómez, A., Kennedy, A. R., and Hevia, E. (2016). Exploiting deep eutectic solvents and organolithium reagent partnerships: chemoselective ultrafast addition to imines and quinolines under aerobic ambient temperature conditions. Angew. Chem. Int. Ed. Engl. 55, 16145-16148. doi: 10.1002/anie.201609929

Watanabe, K., Yamagiwa, N., and Torisawa, Y. (2007). Cyclopentyl methyl ether as a new and alternative process solvent. Org. Process Res. Dev. 11, 251-258. doi: 10.1021/op0680136

Wolfe, J. P., Wagaw, S., Marcoux, J.-F., and Buchwald, S. L. (1998). Rational development of practical catalysts for aromatic carbon-nitrogen bond formation. Acc. Chem. Res. 31, 805-818. doi: 10.1021/ar9600650

Zhang, Q., de Oliveira Vigier, K., Royer, S., and Jérôme, F. (2012a). Deep eutectic solvents: syntheses, properties and applications. Chem. Soc. Rev. 41, 7108-7146. doi: $10.1039 / \mathrm{c} 2 \mathrm{cs} 35178 \mathrm{a}$

Zhang, Y., Yang, X., Yao, Q., and Ma, D. (2012b). CuI/DMPAO-catalyzed $\mathrm{N}$-arylation of acyclic secondary amines. Org. Lett. 14, 3056-3059. doi: 10.1021/ol301135c

Zhou, W., Fan, M., Yin, J., Jiang, Y., and Ma, D. (2015). CuI/oxalic diamide catalyzed coupling reaction of (hetero)aryl chlorides and amines. J. Am. Chem. Soc. 137, 11942-11945. doi: 10.1021/jacs.5b08411

Conflict of Interest: The authors declare that the research was conducted in the absence of any commercial or financial relationships that could be construed as a potential conflict of interest.

Copyright (C) 2019 Quivelli, Vitale, Perna and Capriati. This is an open-access article distributed under the terms of the Creative Commons Attribution License (CC BY). The use, distribution or reproduction in other forums is permitted, provided the original author(s) and the copyright owner(s) are credited and that the original publication in this journal is cited, in accordance with accepted academic practice. No use, distribution or reproduction is permitted which does not comply with these terms. 